\title{
ON MODAL EXTENSIONS OF PRODUCT FUZZY LOGIC
}

\author{
AMANDA VIDAL, FRANCESC ESTEVA AND LLUIS GODO \\ Artificial Intelligence Research Institute (IIIA - CSIC) \\ Campus UAB, 08193 Bellaterra, Spain \\ \{amanda,esteva,godo\}@iiia.csic.es
}

\begin{abstract}
In this paper we study modal extensions of Product fuzzy logic with both relational semantics based on Kripke structures with crisp accessibility relations and algebraic semantics, when the underlying product fuzzy logic is expanded with truth-constants, the $\Delta$ operator and with two infinitary inference rules. We provide completeness results for both kinds of semantics. Finally, we also consider a generalization of possibilistic logic evaluated over product algebras.
\end{abstract}

\section{INTRODUCTION}

The main purpose of this paper is to study some modal extensions (with generalized $\square$ and $\diamond$ modalities) of the Product fuzzy logic. Modal extensions of main systems of mathematical fuzzy logic have appeared in the literature, both as technical contributions and proposing practical motivations for them. We focus on the study of modal extensions of many-valued logics in the sense of Hájek in [Háj98]: these logics appear as a suitable framework to formalize reasoning under vagueness, and the modalities address the logical formalization of notions such as belief, uncertainty, knowledge, preference, time, etc. Several papers have been published on this topic treating different logics: [CR10, CR15, CMRR13] present results concerning different modal extensions of Gödel fuzzy logic, [BEGR11] is a systematic study of modal logics over finite residuated lattices and [HT13] researches the modal extensions of Łukasiewicz and finitely-valued Łukasiewicz logics for a crisp accessibility relation semantics.

However, the study of modal extensions over the Product fuzzy logic $\Pi$, with Kripke style semantics based on either crisp or fuzzy accessibility relations, has remained open so far. We consider its study as a crucial step towards a better understanding of the modal extensions of Hájek's Basic fuzzy logic BL and their extensions. Here, we present some results that partially fill this gap, namely by providing an axiomatisation of the minimal local and global modal logics that arise from Kripke models with worlds evaluating formulas over the canonical standard product algebra and with a crisp accessibility relation. We also explore an algebraic semantics for these logics and how it relates to the relational semantics.

It is worth noticing that the underlying propositional logic we use is not the plain Product logic but its expansion with rational constants, the Baaz-Monteiro $\Delta$ operator and with two additional infinitary inference rules, that make the resulting logic strongly standard complete (i.e. with respect to the semantics defined by the standard product algebra on the real unit interval $[0,1])$. We have two reasons to do this, one for considering the addition of truth constants and another for the addition of the $\Delta$ operator and the two infinitary rules. First, truth constants play a fundamental role in our approach to the proof of completeness of the proposed axiomatization: whereas in the standard Gödel algebra, one can always differentiate two different elements by an endomorphism (sending the greatest to 1 and the other to some value less than 1) and in Eukasiewicz logic there are terms that can play this role (see e.g. [Ost88]), in Product logic we have to resort to truth constants to be able to discriminate among different elements in the standard algebra. Second, we need to have strong completeness of the underlying propositional logic (this is also the case for the modal extensions of Gödel and Lukasiewicz logics), even if the modal logic is defined as finitary; in 
our case of Product logic with rational constants, strong completeness can be achieved by extending the language with the $\Delta$ operator and adding to the axiomatic system two infinitary rules.

The paper is structured as follows. To make to the paper self contained as much as possible, Section 2 gathers some preliminaries about Product fuzzy logic as well as some basic notions about abstract algebraic logic. Section 3 presents some results concerning strong completeness of the infinitary Product logic with rational truth constants and the Monteiro-Baaz $\Delta$ operator, extending and providing alternative proofs to some related contributions found in [Cin04] and [Mon06]. The main result presented in this section is the strong standard completeness of our proposed axiomatization, namely Theorem 3.10. The approach followed to prove this result is more algebraic than the one done by Cintula: we study the class of algebras for this logic and present some results concerning the behaviour of the filters of the logic over them (Lemma 3.5), that will give as corollaries the Linear Extension Property of the logic and the strong completeness with respect to the canonical standard product algebra. In Section 4 we expand the language with modal operators $\square$ and $\diamond$, and we present two axiomatizations (Definitions 4.2 and 4.3). The main results from this section are Theorems 4.11 and 4.12, that prove the strong completeness of the previous axiomatizations with respect to the local and global logical consequences induced by Kripke structures with crisp accessibility relations (described in Definition 4.1).

Section 5 deals with the algebraic semantics of these logics, defining the class of modal product algebras associated with them, studying its classification within the Leibniz hierarchy and presenting completeness theorems and other characterization results. The most remarkable results at this respect are the completeness of the global logic with respect to its algebraic class in the usual sense -with models formed by algebras of the class and $\{1\}$ as the designated truth values-, (Theorem 5.4), and, in contra position, the non-algebraiazbility of the local modal logic, showed in Lemma lemma:nonAlg (expected from the behaviour of the classical (local) modal logic, but nevertheless, non-trivial because of the infinitary behaviour of our logics) and the subsequent characterization of its reduced models (Theorem 5.8). Moreover, we prove in Theorem 5.9 that the local and global logics are not complete with respect to linearly ordered modal algebras. This section also provides details on the deep relationship between the Kripke semantics and the algebraic semantics for these modal logics. First, this allows to present an algebraic completeness result for the local modal logic resorting to the order preserving logics (see Theorem 5.13). Moreover, we have developed the mappings between (product) modal algebras and Kripke models, getting several interesting results on the behaviour of the deductions when applying these mappings $(5.22,5.21,5.20)$, that leave as open work that of studying the duality between these two classes. Section 6 concludes the study of the product modal logics with several further results: in Section 6.1 results concerning the interdefinability of the modal operators are proven (see Theorem 6.2); in Section 6.2, we consider axiomatic extensions with some of the most common axioms used in normal modal logics $(\mathrm{D}, \mathrm{T}, 4,5)$, and check completeness with respect to their respective classes of product Kripke models (Theorem 6.5); finally, in Section 6.3, we consider a possibilistic extension of the fuzzy modal product logic and study its axiomatization and completeness. We end in Section 7 with some final conclusions and proposing some interesting open problems. For the sake of readability, the proofs of some theorems have been presented in an annex for the interested reader. They are rather technical proofs that are not essential for the comprehension of the article.

Notation coventions. The main conventions about notation that we will use throughout this paper are as follows. Classes of algebras will be denoted as $\mathbb{C}, \mathbb{D}, \ldots$; algebras as $\boldsymbol{A}, \boldsymbol{B}, \ldots$; sets (and so, algebra universes) as $A, B, \ldots$ and elements of a set (algebra) as $a, b, \ldots$ In particular, for a logic $\mathcal{L}, \boldsymbol{F} \boldsymbol{m}_{\mathcal{L}}$ will denote the algebra of formulas built from a denumerable set of propositional variables on the language of the logic. If not specified otherwise, $x, y, \ldots$ will denote propositional variables, and $\varphi, \psi, \ldots$ formulas (over the corresponding language). Kripke models will be denoted by $\mathfrak{M}, \mathfrak{G}, \ldots$ Finally, given two sets $A, B$ we will write $A^{B}$ to denote the set of mappings from $B$ to 
$A, f, g, \ldots$ to name the elements of this set, and for $f \in A^{B}$, we will also write $\left[a \mapsto f_{a}\right]$ to denote it.

\section{Preliminaries}

In this section we provide some background definitions and existing results that are needed in order to understand the paper without continuously resorting to external sources. First, we overview Product fuzzy logic, which will be the starting point of our study. Second, we provide a refresher of several notions and results from abstract algebraic logic, that will be used in Sections 3 and 5 .

2.1. Product fuzzy logic. This article is settled over the so-called Product fuzzy logic $\Pi$ presented by Hájek et al. in [HGE96] as a propositional logic over the language $\mathfrak{L}=\{\&, \rightarrow, \overline{0}\}$. Other usual connectives can be defined from these: $\overline{1}:=\varphi \rightarrow \varphi, \neg \varphi:=\varphi \rightarrow \overline{0}, \varphi \wedge \psi:=\varphi \&(\varphi \rightarrow \psi)$ and $\varphi \vee \psi:=((\varphi \rightarrow \psi) \rightarrow \psi) \wedge((\psi \rightarrow \varphi) \rightarrow \varphi)$. Several equivalent axiomatizations of $\Pi$ have been presented, shortening the original one provided in [HGE96] (Hájek in [Háj98], Cintula in [Cin01] and Montagna et al. in [MNH06]). We will consider this last one:

(A1) $(\varphi \rightarrow \psi) \rightarrow((\psi \rightarrow \chi) \rightarrow(\varphi \rightarrow \chi))$

(A2) $\varphi \& \psi \rightarrow \varphi$

(A3) $\varphi \& \psi \rightarrow \psi \& \varphi$

(A4) $\varphi \&(\varphi \rightarrow \psi) \rightarrow(\psi \&(\psi \rightarrow \varphi))$

(A5a) $(\varphi \rightarrow(\psi \rightarrow \chi)) \rightarrow((\varphi \& \psi) \rightarrow \chi)$

$(\mathrm{A} 5 \mathrm{~b})((\varphi \& \psi) \rightarrow \chi) \rightarrow(\varphi \rightarrow(\psi \rightarrow \chi))$

(A6) $((\varphi \rightarrow \psi) \rightarrow \chi) \rightarrow(((\psi \rightarrow \varphi) \rightarrow \chi) \rightarrow \chi)$

(II) $\neg \varphi \vee((\varphi \rightarrow(\varphi \& \psi)) \rightarrow \psi)$

The only inference rule of $\Pi$ is Modus Ponens $(M P)$.

It is clear that $(A 1)$ - $(A 6)$ together with $(M P)$ define Hájek's Basic Fuzzy Logic $B L$ [Háj98], whose extension under the adequate axioms provides, respectively, axiomatizations of Gödel-Dummet, Eukasiewicz, and Product logics. The finitary notion of proof in $\Pi$ is the usual one, and for an arbitrary set of formulas $\Gamma \cup\{\varphi\}$ in the language $\mathfrak{L}$, we shall write $\Gamma \vdash_{\Pi} \varphi$ to denote that there exists a finite $\Gamma_{0} \subseteq \Gamma$ and a proof of $\varphi$ from $\Gamma_{0}$.

The algebraic companion of $\Pi$ is the variety of product algebras, i.e. algebras of the form $\boldsymbol{A}=\langle A, \odot, \Rightarrow, \wedge, \vee, 0,1\rangle$ which are commutative, integral and bounded residuated lattices satisfying the following conditions:

- $(x \Rightarrow y) \vee(y \Rightarrow x)=1$;

- $\neg \neg x \odot(x \odot z \Rightarrow y \odot z) \leqslant x \Rightarrow y$;

- $x \wedge \neg x=0$;

- $\left\{\begin{array}{l}x \odot(y \vee z) \leqslant(x \odot y) \vee(x \odot z) \\ x \odot(y \wedge z) \geqslant(x \odot y) \wedge(x \odot z)\end{array}\right.$

where $\neg x:=x \Rightarrow 0$.

An evaluation of formulas $h$ on a product algebra $\boldsymbol{A}$ is a mapping from the set of formulas into $A$ satisfying $h(\overline{0})=0, h(\varphi \& \psi)=h(\varphi) \odot h(\psi)$ and $h(\varphi \rightarrow \psi)=h(\varphi) \Rightarrow h(\psi)$.

As proven in [HGE96], Product logic enjoys finite strong completeness with respect to the standard Product Algebra $[0,1]_{\Pi}=\left\langle[0,1], \cdot, \Rightarrow_{\Pi}, 0\right\rangle$ (where $x \cdot y$ is the usual product on $[0,1]$ and $x \Rightarrow_{\Pi} y$ equals 1 if $x \leqslant y$ and $y / x$ otherwise). That is to say, for any finite set of formulas $\Gamma \cup\{\varphi\} \subseteq F m$, $\Gamma \vdash_{\Pi} \varphi$ holds if and only if for any evaluation of formulas $h$ into $[0,1]_{\Pi}$, if $h([\Gamma]) \subseteq\{1\}$ then $h(\varphi)=1$.

Unless specified otherwise, we will write $\Gamma=_{\boldsymbol{A}} \varphi$ for an arbitrary algebra $\boldsymbol{A}$ whenever for any evaluation of formulas $h$ into $\boldsymbol{A}$, if $h([\Gamma]) \subseteq\{1\}$ then $h(\varphi)=1$.

Extending this work, in $\left[\mathrm{SCE}^{+} 06\right]$ the authors studied Product logic over a language expanded with countably many truth constants, one $\bar{c}$ for each rational $c \in[0,1]$. They also included in their 
study the expansion of the language with the Monteiro-Baaz unary operator $\Delta$, whose interpretation in a product chain is the function $\delta$ defined by $\delta(1)=1, \delta(x)=0$ for all $x<1$. They propose an axiomatization that extends $\Pi$ with book-keeping axioms that model the behaviour of the rational constant symbols and some new axioms and an inference rule regarding the $\Delta$ operation. We will denote by $\Pi_{\Delta}^{c}$ the axiomatic system resulting from adding to $\Pi$ the following axioms and rule:
$\left(\mathrm{A}_{\Delta} 1\right) \Delta \varphi \vee \neg \Delta \varphi$
$\left(\mathrm{A}_{\Delta} 2\right) \Delta(\varphi \vee \psi) \rightarrow(\Delta \varphi \vee \Delta \psi)$
$\left(\mathrm{A}_{\Delta} 3\right) \Delta \varphi \rightarrow \varphi$
$\left(\mathrm{A}_{\Delta} 4\right) \Delta \varphi \rightarrow \Delta \Delta \varphi$
$\left(\mathrm{A}_{\Delta} 5\right) \Delta(\varphi \rightarrow \psi) \rightarrow(\Delta \varphi \rightarrow \Delta \psi)$
$\left(\mathbf{G}_{\Delta}\right) \varphi \vdash \Delta \varphi$

$\left(\mathrm{A}_{\mathrm{c}} 1\right) \bar{c} \& \bar{d} \leftrightarrow \overline{c \cdot d}$

$\left(\mathrm{A}_{\mathrm{c}} 2\right)(\bar{c} \rightarrow \bar{d}) \leftrightarrow \overline{c \Rightarrow_{\Pi} d}$

$\left(\mathrm{A}_{\mathrm{c}} 3\right) \neg \Delta \bar{c}$ for any $c<1$;

Again, the notion of proof in $\Pi_{\Delta}^{c}$ is the usual finitary one. The product algebra with universe $[0,1]$, with rational constants interpreted as its name and with $\Delta$ operator will be called canonical standard product algebra. Given that this one will be the only product algebra referred to in the following sections there is no place to confusion, and for the sake of readability we will abuse notation and denote the canonical standard product algebra by $[0,1]_{\Pi}$.

$\Pi_{\Delta}^{c}$ was proven in $\left[\mathrm{SCE}^{+} 06\right]$ to be finitely strong complete with respect to the canonical standard product algebra (i.e. $[0,1]_{\Pi}$ generates the variety of product algebras with rational constants and

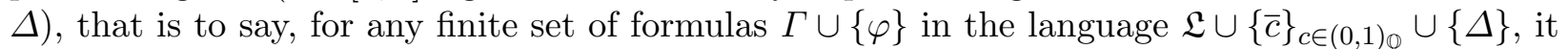
hods that $\Gamma \vdash_{\Pi_{\Delta}^{c}} \varphi$ if and only if $\Gamma \models_{[0,1]_{\Pi}} \varphi$.

2.2. Abstract algebraic logic. The study of the algebraization of a certain logic, and in particular, its classification within the Leibniz hierarchy determines the extent to which that logic may be studied using known algebraic methods and techniques. Over the last 30 years, a powerful arsenal of results that study the different levels of the Leibniz hierarchy has been obtained, and they can be naturally used to obtain an exhaustive characterization of a logic once it has been assigned to a level of this hierarchy.

We will recall here some basic notions that will allow us to classify the logics defined in Section 4 in the Leibniz hierarchy and to study their algebraic semantics. Most of the definitions and results recalled here are folklore; for a systematic exposition of these see for instance [BP86], [BP89], [Cze01] and [FJP03].

Recall that, given a class of algebras $\mathbb{K}$ and an algebra $\boldsymbol{A}$, a congruence $\theta$ of $\boldsymbol{A}$ is a $\mathbb{K}$-congruence, or a congruence of $\boldsymbol{A}$ relative to $\mathbb{K}$, when $\boldsymbol{A} / \theta \in \mathbb{K}$. The class of congruences of $\boldsymbol{A}$ relative to $\mathbb{K}$ is denoted by $C o_{\mathbb{K}} \boldsymbol{A}$. If $\mathbb{K}$ is closed under subdirect products, the class of congruences of any algebra relative to $\mathbb{K}$ forms a complete lattice.

In this work, we will restrict ourselves to the case when $\mathbb{K}$ is a generalised quasi-variety, that is, a class of algebras axiomatized with generalised quasi-equations (quasi-equations with a possible infinite set of premises written with countably many variables). These are, in particular, $\mathbb{I S P}_{S}$ classes (closed under isomorphism, subalgebras and subdirect products), so it will be correct to talk about the lattice of congruences relative to $\mathbb{K}$.

Definition 2.1. Let $\langle\boldsymbol{A}, D\rangle$ be a logical matrix, i.e., $\boldsymbol{A}$ is an algebra and $D \subseteq A$. The Leibniz congruence of $\langle\boldsymbol{A}, D\rangle$, denoted $\boldsymbol{\Omega}^{\boldsymbol{A}} D$, is the largest congruence of $\boldsymbol{A}$ compatible with $D$ (i.e., if $a \in D$ and $a \equiv b\left(\boldsymbol{\Omega}^{\boldsymbol{A}} D\right)$ then $\left.b \in D\right)$.

Given two algebras $\boldsymbol{A}$ and $\boldsymbol{B}$ of the same type, we will denote by $\operatorname{Hom}(\boldsymbol{A}, \boldsymbol{B})$ the set of homomorphisms from $\boldsymbol{A}$ to $\boldsymbol{B}$. Then, for a logic $\mathcal{L}$ and an algebra $\boldsymbol{A},{ }^{1}$ a set $F \subseteq A$ is called a filter of $\mathcal{L}$ (or a $\mathcal{L}$-filter) on $\boldsymbol{A}$ whenever for any $\Gamma \cup\{\varphi\} \subseteq F m_{\mathcal{L}}$ (that is, formulas in the language $\mathcal{L})$ and $h \in H \operatorname{Hom}\left(\boldsymbol{F} \boldsymbol{m}_{\mathcal{L}}, \boldsymbol{A}\right)$, if $\Gamma \vdash_{\mathcal{L}} \varphi$ and $h \Gamma \subseteq F$, then $h \varphi \in F$. The set of all $\mathcal{L}$-filters on the

\footnotetext{
${ }^{1}$ We will always assume that the type of the algebras is the one of the logic.
} 
algebra $\boldsymbol{A}$ is denoted by $\mathcal{F} i_{\mathcal{L}} \boldsymbol{A}$ and the class of models of $\mathcal{L}, \operatorname{Mod} \mathcal{L}$, will consist on the pairs of the form $\langle\boldsymbol{A}, F\rangle$ where $\boldsymbol{A}$ is an algebra of the type of $\mathcal{L}$ and $F \in \mathcal{F} i_{\mathcal{L}} \boldsymbol{A}$.

It is a general fact that any logic $\mathcal{L}$ is strongly complete with respect to its class of reduced models, $\operatorname{Mod}^{*} \mathcal{L}$, the models of $\mathcal{L}$ for which $\Omega^{A} F=\operatorname{Id}_{A}$. We will denote by $A l g^{*} \mathcal{L}$ the class of algebras $\boldsymbol{A}$ for which there exists $F \in \mathcal{F} i_{\mathcal{L}} \boldsymbol{A}$ such that $\langle\boldsymbol{A}, F\rangle \in \operatorname{Mod}^{*} \mathcal{L}$.

Lemma 2.2. Let $\mathcal{L}$ be a logic and $\boldsymbol{\Delta}(x, y)$ a set of formulas with only $x$ and $y$ as variables. The following conditions are equivalent:

(1) $\boldsymbol{\Delta}(x, y)$ satisfies the following conditions:

$(\mathrm{R}) \vdash_{\mathcal{L}} \boldsymbol{\Delta}(x, x)$

(MP) $x, \boldsymbol{\Delta}(x, y) \vdash_{\mathcal{L}} y$

(Re) $\bigcup_{i<n} \boldsymbol{\Delta}\left(x_{i}, y_{i}\right) \vdash_{\mathcal{L}} \boldsymbol{\Delta}\left(\lambda\left(x_{0} \ldots x_{n-1}\right), \lambda\left(y_{0} \ldots y_{n-1}\right)\right)$ for each $\lambda$ in the language of $\mathcal{L}$, with $n=\operatorname{ar} \lambda$.

(2) For all $\langle\boldsymbol{A}, F\rangle \in M o d \mathcal{L}, a \equiv b\left(\boldsymbol{\Omega}^{\boldsymbol{A}} F\right)$ if and only if $\boldsymbol{\Delta}^{\boldsymbol{A}}(a, b) \subseteq F$.

A $\operatorname{logic} \mathcal{L}$ for which there exists a set of formulas $\boldsymbol{\Delta}(x, y)$ that satisfies any of the previous equivalent properties is said to be equivalential with congruence formulas $\boldsymbol{\Delta}(x, y){ }^{2}$

Lemma 2.3. Let $\mathcal{L}$ be an equivalential logic. Then for every algebra $\boldsymbol{A}$, the Leibniz operator $\boldsymbol{\Omega}^{\boldsymbol{A}}$ is monotonic over the $\mathcal{L}$-filters on $\boldsymbol{A}$.

Definition 2.4. A logic $\mathcal{L}$ is algebraizable when there is a class $\mathbb{K}$ of algebras, a set of formulas $\boldsymbol{\Delta}(x, y)$ in at most two variables (congruence formulas), and a set of equations $\mathbf{E}(x)$ in at most one variable (defining equations) such that for all $\Gamma \cup\{\varphi\} \subseteq F m_{\mathcal{L}}$ the following conditions hold:

- $\Gamma \vdash_{\mathcal{L}} \varphi$ if and only if $\mathbf{E}(\Gamma) \models_{\mathbb{K}} \mathbf{E}(\varphi)$;

- $x \approx y \models_{\mathbb{K}} \mathbf{E}(\boldsymbol{\Delta}(x, y))$ and $\mathbf{E}(\boldsymbol{\Delta}(x, y)) \models_{\mathbb{K}} x \approx y$.

where for an arbitrary set of formulas $X$, we write $\mathbf{E}(X)$ to denote the set of equations $\bigcup_{x \in X} \mathbf{E}(x)$, and for $E 1 \cup E 2$ a set of equations in the language of $\mathcal{L}$ (with at most $\aleph_{0}$ variables), $E 1 \models \mathbb{K} E 2$ if and only if for all $\boldsymbol{A} \in \mathbb{K}$ and for all $h \in \operatorname{Hom}\left(\boldsymbol{F m}_{\mathcal{L}}, \boldsymbol{A}\right)$, if $h(\varepsilon)=h(\delta)$ for every $\varepsilon \approx \delta \in E 1$, then $h(\alpha)=h(\beta)$ for all $\alpha \approx \beta \in E 2$.

In such a case, $\mathbb{K}$ is called an algebraic semantics of $\mathcal{L}$. The greatest algebraic semantics $\mathbb{K}$ of $\log L$ (ordered by the inclusion) is called equivalent algebraic semantics and it coincides with the quasi-variety generated by any algebraic semantics of $\mathcal{L}$.

We will finish this brief reminder on abstract algebraic logic by stating a very important result that deeply relates the filters of an algebraizable logic and the congruences relative to its class of algebras.

Theorem 2.5 (Isomorphism Theorem). Let $\mathcal{L}$ be a logic and $\mathbb{K}$ a generalized quasi-variety. $\mathcal{L}$ is algebraizable with equivalent algebraic semantics $\mathbb{K}$ if and only if in any algebra $\boldsymbol{A}$, the Leibniz operator is an isomorphism between the lattices $\mathcal{F} i_{\mathcal{L}} \boldsymbol{A}$ and $C_{o_{\mathbb{K}}} \boldsymbol{A}$ that commutes with endomorphisms.

As a last remark, relying in this isomorphism, if $\mathcal{L}$ is an algebraizable logic with algebraic semantics $\mathbb{K}$ we will simplify the notation by writing $\boldsymbol{A} / F$ instead of $\boldsymbol{A} / \boldsymbol{\Omega}^{\boldsymbol{A}} F$, whenever $\boldsymbol{A} \in \mathbb{K}$ and $F$ is an $\mathcal{L}$-filter on $\boldsymbol{A}$.

\section{Infinitary Product LOGiC With RATIONAL CONSTANTS AND $\Delta$}

In the previous section, we remarked the fact that both $\Pi$ and $\Pi_{\Delta}^{c}$ enjoyed finite strong completeness. However, for reasons that were commented in the introduction and that will become clearer in Section 4, we are interested in defining a logic that is complete with respect to arbitrary logical consequences (in the sense that infinite sets of premises are considered too) over the canonical

\footnotetext{
${ }^{2}$ Do not confuse this $\boldsymbol{\Delta}$ binary function that provides a set of equation with the $\Delta$ Baaz-Monterio unary operator of the logic.
} 
standard product algebra. A simple counterexample shows that neither $\Pi$ nor $\Pi_{\Delta}^{c}$ are strongly standard complete.

Example 3.1. Let $p, q$ be propositional variables and let $\Gamma=\left\{p \rightarrow q^{n} \mid n \in \mathbb{N}\right\}$, where $q^{n}$ is the abbreviation of $q \& \stackrel{n}{\text {. } ~ \& ~} q$. For any evaluation $v$ on $[0,1]_{\Pi}$, if $v\left(p \rightarrow q^{n}\right)=1$ for all $n \in \mathbb{N}$, then either $v(p)=0$ or $v(q)=1$, which is equivalent to $v(\neg p \vee q)=1$, i.e., $\Gamma \models_{[0,1]_{\Pi}} \neg p \vee q$. However, it is clear that for any finite subset $\Gamma_{0}$ of $\Gamma, \Gamma_{0} \vDash_{[0,1]_{\Pi}} \neg p \vee q$. Hence $\models_{[0,1]_{\Pi}}$ is infinitary, while $\vdash_{\Pi}$ is finitary.

In [Mon06], Montagna deals with the problem of the infinitary character of the semantical consequence relation of logics based on continuous t-norms, and obtains a general result for the family of BL logics. He defines a unary operator $*$, called storage operator, over any $B L$ algebra, with $* x$ being the maximum idempotent element smaller or equal to $x$, and studies the semilinear expansion of any BL logic with storage operator ${ }^{3}$ and the infinitary rule

$$
\frac{\chi \vee\left(\varphi \rightarrow \psi^{k}\right) \text { for all } k \in \omega}{\chi \vee\left(\varphi \rightarrow \psi^{*}\right)}
$$

where, as usual, $\psi^{k}$ denotes $\psi \& . k$. \& $\psi$. This expansion is proved to be strongly complete (for infinite theories) with respect to the corresponding class of standard BL-chains. In particular, it can be seen that for Product logic, the $*$ operator coincides with the Monteiro-Baaz operator $\Delta$ in $[0,1]$.

On the other hand, when working with logics extended with rational constants some results have been obtained in the frame of rational Pavelka-like logics by Cintula in his $\mathrm{PhD}$ dissertation [Cin04] and in a very recent paper [Cin14]. In particular, in the case of the Product logic with $\Delta$ operator, he proved that the logic $\Pi^{\infty}$ defined from $\Pi_{\Delta}^{c}$ by the addition of two infinitary rules that manage the behaviour of the constants in the discontinuity points of $\Delta(1)$ and $\rightarrow(0)$ is Pavelka complete, i.e., for an arbitrary set of formulas $\Gamma \cup\{\varphi\}$, the truth degree of $\varphi$ over $\Gamma$, defined as $\|\varphi\|_{\Gamma}=\inf \left\{e(\varphi): e \in \operatorname{Hom}\left(\boldsymbol{F m}_{\Pi_{\Pi} \infty},[0,1]_{\Pi}\right)\right.$ such that $\left.e([\Gamma]) \subseteq\{1\}\right\}$, coincides with the provability degree of $\varphi$ over $\Gamma$, defined as $|\varphi|_{\Gamma}=\sup \left\{c \mid \Gamma \vdash_{\Pi^{\infty}} \bar{c} \rightarrow \varphi\right\}$.

Definition 3.2. $\Pi^{\infty}$ is the infinitary logic obtained by extending $\Pi_{\Delta}^{c}$ with the following two infinitary rules:

$$
\left(\mathrm{R}_{\Delta}\right) \frac{\bar{c} \rightarrow \varphi, \text { for all } c \in(0,1)_{\mathbb{Q}}}{\varphi} \quad\left(\mathrm{R}_{\rightarrow}\right) \frac{\varphi \rightarrow \bar{c}, \text { for all } c \in(0,1)_{\mathbb{Q}}}{\neg \varphi} ;
$$

The notion of infinitary deduction for these kinds of logics is worth to be recalled (cf. [Cin04, Def. 2.1.10]).

Definition 3.3. Let $\Gamma \cup\{\varphi\} \subseteq F m_{\Pi \infty}$. A proof of $\varphi$ from $\Gamma$ in $\Pi^{\infty}$ is a well-founded tree (with possibly infinite width and finite depth) labelled by formulae such that

- The root is labelled by $\varphi$, and the leaves are axioms of $\Pi^{\infty}$ or elements from $\Gamma$.

- For each intermediate node $\psi$ with $\Sigma_{\psi}$ being its successors in the tree, there is a rule $\Sigma_{\psi} \vdash \psi$ in $\Pi^{\infty}$.

As usual, we write $\Gamma \vdash_{\Pi^{\infty}} \varphi$ whenever there exists a proof of $\varphi$ from $\Gamma$ in $\Pi^{\infty}$.

Note that the $\Delta$-deduction theorem holds for $\Pi^{\infty}: \Gamma \cup\{\varphi\} \vdash_{\Pi^{\infty}} \psi$ iff $\Gamma \vdash_{\Pi^{\infty}} \Delta \varphi \rightarrow \psi$, see e.g. [VEG14]. Actually in [VEG14] preliminary results about the logic $\Pi^{\infty}$ have been presented.

It is clear that, enjoying Pavelka style completeness and having the infinitary rule $\left(R_{\Delta}\right), \Pi^{\infty}$ also enjoys strong completeness with respect to $[0,1]_{\Pi}$. However, it is also possible to follow a different path, namely an algebraic one, to prove the strong standard completeness of $\Pi^{\infty}$. That is, first to characterize and study the algebraic companion of this logic and prove strong completeness with respect to linearly ordered algebras from this class, and then, gaining inspiration from ideas

\footnotetext{
${ }^{3}$ In [Mon06], these logics correspond to representable BL-algebras with storage.
} 
in [Mon06], to prove strong completeness as well with respect to the canonical standard product algebra. The advantage of this latter path, that we will explore in the rest of this section, is to obtain a deeper insight into the logic through its algebraic counterpart.

Before characterizing the algebraic companion of $\Pi^{\infty}$, notice that it is easy to prove that the two new infinitary rules hold one of the characteristics of semilinear logics: they are closed under the disjunction operation. That is to say, the following are valid deductions in $\Pi^{\infty}$ :

$$
\left(\mathrm{R}_{\Delta}^{\prime}\right) \frac{\{\chi \vee(\bar{c} \rightarrow \varphi)\}_{c \in(0,1)_{\mathbb{Q}}}}{\chi \vee \varphi} \quad\left(\mathrm{R}_{\rightarrow}^{\prime}\right) \frac{\{\chi \vee(\varphi \rightarrow \bar{c})\}_{c \in(0,1)_{\mathbb{Q}}}}{\chi \vee \neg \varphi}
$$

Facing the algebraic study of $\Pi^{\infty}$, it is immediate to check that it is a Raisowa-implicative logic, and thus algebraizable in the sense of Blok and Pigozzi [BP89]. Its algebraic companion coincides with the class $\mathbb{P}^{\infty}$ of $\Pi^{\infty}$-algebras $\boldsymbol{A}=\left\langle A, \odot, \Rightarrow, \Delta,\left\{c^{\boldsymbol{A}}\right\}_{c \in[0,1]_{\mathbb{Q}}}\right\rangle$ where:

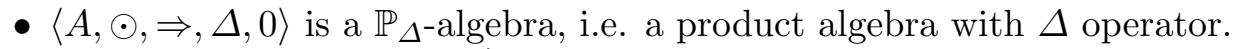

- The rational constants $\left\{c^{\boldsymbol{A}}\right\}$ form a subalgebra isomorphic to $[0,1]_{\mathbb{Q}}\left(\right.$ as $\mathbb{P}_{\Delta}$-algebras) such that for each $c, d \in[0,1]_{\mathbb{Q}}$ and $x \in A$ the following equations and generalised quasi-equations hold:

$$
\begin{aligned}
& -c^{\boldsymbol{A}} \odot d^{\boldsymbol{A}}=(c \cdot d)^{\boldsymbol{A}} \\
& -c^{\boldsymbol{A}} \Rightarrow d^{\boldsymbol{A}}=\min \left\{1,(d / c)^{\boldsymbol{A}}\right\} \\
& -\Delta c^{\boldsymbol{A}}=0 \text { for } c<1 \\
& \text { - If } x \geqslant c^{\boldsymbol{A}} \text { for all } c \in(0,1)_{\mathbb{Q}} \text { then } x=1 \\
& \text { - If } x \leqslant c^{\boldsymbol{A}} \text { for all } c \in(0,1)_{\mathbb{Q}} \text { then } x=0 .
\end{aligned}
$$

Since $\Pi^{\infty}$ is implicative, it enjoys the following form of completeness: For any $\Gamma \cup\{\varphi\} \subseteq F m_{\Pi^{\infty}}$,

$$
\Gamma \vdash_{\Pi^{\infty}} \varphi \text { iff } h([\Gamma]) \subseteq\{1\} \text { implies } h(\varphi)=1 \text { for all } h \in H o m\left(\boldsymbol{F m}_{\boldsymbol{\Pi}^{\infty}}, \boldsymbol{A}\right) \text { and all } \boldsymbol{A} \in \mathbb{P}^{\infty} .
$$

The class $\mathbb{P}^{\infty}$ is a generalised quasi-variety, and it can be proven to be not a quasi-variety, since it is not closed under ultraproducts.

Example 3.4. Let $U$ be the set of cofinite subsets of $\omega$, and consider the ultraproduct $\prod_{n \in \omega}[0,1]_{\Pi} / U$ (i.e., for $a, b \in \prod_{n \in \omega}[0,1]_{\Pi}, a \sim_{U} b \Leftrightarrow\{n \in \omega: a[n] \neq b[n]\}$ is a finite set). Let $p \in \prod_{n \in \omega}^{n \in \omega}[0,1]_{\Pi}$ be the element defined by $p[n]=\frac{2^{n}-1}{2^{n}}$ for each $n \in \omega$. It is an strictly increasing series, and given that $\lim _{n \rightarrow \infty} \frac{2^{n}-1}{2^{n}}=1$, for any $c \in(0,1)_{\mathbb{Q}}$ there is some $s \in \omega$ such that $\frac{2^{s}-1}{2^{s}} \geqslant c$. Denote by $c^{\omega}$ the element such that $c^{\omega}[n]=c$ for each $n \in \omega$. Then $\left(c^{\omega} \rightarrow p\right)[i]=1$ for all $i \geqslant s$ and thus, by definition, $c^{\omega} \rightarrow p \sim 1$ for all $c \in(0,1)_{\mathbb{Q}}$. Since the constants in this ultraproduct are by definition the equivalence classes of $c^{\omega}$ for each $c \in(0,1)_{\mathbb{Q}}$, it holds that $c^{\omega} \rightarrow p=1$ for each $c \in(0,1)_{\mathbb{Q}}$. However, $p[n]<1$ for each $n \in \omega$, and thus it is not true that $p=1$ in $\prod_{n \in \omega}[0,1]_{\Pi} / U$, getting that $\prod_{n \in \omega}[0,1]_{\Pi} / U \notin \mathbb{P}^{\infty}$.

Completeness of $\Pi^{\infty}$ with respect to linearly ordered algebras of $\mathbb{P}^{\infty}$ can be deduced from the fact that for any set of formulas $\Gamma \cup\{\alpha\}$ such that $\Gamma \forall_{\Pi^{\infty}} \alpha, \Gamma$ can be extended to a linear $\Pi^{\infty}$-theory $\Gamma^{+}$(i.e., one where for any formulas $\varphi, \psi$, either $\varphi \rightarrow \psi \in \Gamma^{+}$or $\psi \rightarrow \varphi \in \Gamma^{+}$) for which still $\Gamma^{+} \forall \Pi_{\infty} \alpha$, a result that can be found implicit within the proof of Lemma 3.4.25 of Cintula's doctoral dissertation [Cin04]. ${ }^{4}$

For our purposes, it is interesting to follow an alternative algebraic approach, characterizing each filter of the logic over an algebra in terms of the linear filters (those for which given two arbitrary

\footnotetext{
${ }^{4}$ Indeed, observe that for such $\alpha$ and $\Gamma^{+}$, the quotient $\boldsymbol{F} \boldsymbol{m}_{\boldsymbol{\Pi}} \infty / \boldsymbol{\Omega} \Gamma^{+}$(i.e., where $\varphi \sim \psi$ when $\varphi \rightarrow \psi$ and $\psi \rightarrow \varphi$ belong to $\Gamma^{+}$) is a $\Pi^{\infty}$-chain. It is immediate then to check that the evaluation into this algebra that maps each formula to its equivalence class sends all the elements from $\Gamma^{+}$to $\{1\}$, and sends $\alpha$ to a value less than 1 (since it does not follow from $\Gamma^{+}$).
} 
elements $a, b$ of the algebra, either $a \rightarrow b \in F$ or $b \rightarrow a \in F)$ that contain it. Indeed, observe that for a linear $\Pi^{\infty}$-filter $F$ on a $\Pi^{\infty}$-algebra $\boldsymbol{A}$, the quotient algebra $\boldsymbol{A} / F$ is linearly ordered.

Lemma 3.5. Let $\boldsymbol{A}$ be a $\Pi^{\infty}$-algebra and $F$ a $\Pi^{\infty}$-filter over $\boldsymbol{A}$. Then $F$ is the intersection of all the linear $\Pi^{\infty}$-filters over $\boldsymbol{A}$ that contain it.

Proof. The proof can be found in the Annex.

Strong completeness of $\Pi^{\infty}$ with respect to the linearly ordered $\Pi^{\infty}$-algebras is now a direct corollary from the previous lemma.

Corollary 3.6. For any set of formulas $\Gamma \cup\{\varphi\}$, the following are equivalent:

(1) $\Gamma \vdash_{\Pi \infty} \varphi$;

(2) $\Gamma \models_{C \mathbb{P}^{\infty}} \varphi$, with $C \mathbb{P}^{\infty}$ denoting the class of chains in $\mathbb{P}^{\infty}$.

Proof. From condition (1), if $\Gamma \forall_{\Pi^{\infty}} \varphi$, there is $\boldsymbol{A} \in \mathbb{P}^{\infty}$ and $h$ such that $h([\Gamma])=\{1\}$ and $h(\varphi)<1$. Since $\{1\}$ is a $\Pi^{\infty}$-filter it is the intersection of all linear filters on $\boldsymbol{A}$, and thus there must exist a linear filter $F$ such that $h(\varphi) \notin F$. Then the result follows by observing that $\boldsymbol{A} / F$ is linearly ordered.

From this point, to prove strong standard completeness we gain inspiration in the work of Montagna [Mon06] and extend some of his results to prove that any chain from $\mathbb{P}^{\infty}$ can be completely embedded into the canonical standard algebra. We will be using the notion of archimedean property in product algebras, i.e., for all $0<a, b<1$ there is a positive integer $n$ such that $b^{n}<a$ (where $b^{n}$ denotes the iteration of $b$ with itself under the $\odot$ operation $n$ times). Indeed, Montagna realizes the importance of this property in the standard $B L$ algebras, and his infinitary rule translates it into syntactical terms. In the particular case of product algebras he provides a very neat characterization for it.

Lemma 3.7. ([Mon06, Lemma 10]) Let $\boldsymbol{A}$ be a linearly ordered product algebra. $\boldsymbol{A}$ is archimedean if and only if for any $a, b \in A$ such that $a \leqslant b^{n}$ for all $n$, then $a \leqslant \Delta b$.

When considering a language extended not only with $\Delta$ but also with rational constants, we can check that, whenever the generalized quasiequations $\mathcal{Q}_{\Delta}$ and $\mathcal{Q} \rightarrow$ hold, the last characterization of the archimedean property applies.

Lemma 3.8. Any linearly ordered $\boldsymbol{A} \in \mathbb{P}^{\infty}$ is archimedean.

Proof. Let $a, b \in \boldsymbol{A}$ with $a \leqslant b^{n}$ for all positive integer $n$. We can prove by cases that $a \leqslant \Delta b$.

- If there is $c \in(0,1)_{\mathbb{Q}}$ such that $b \leqslant c^{\boldsymbol{A}}$, then $a \leqslant b^{n} \leqslant\left(c^{\boldsymbol{A}}\right)^{n}$ for all $n$. Moreover $[0,1]_{\mathbb{Q}}$ is archimedean, and so, by axiom $\left(A_{c} 1\right) a \leqslant d^{\boldsymbol{A}}$ for any $d \in(0,1)_{\mathbb{Q}}$. Then, by $\mathcal{Q}_{\rightarrow}, a=0$, and trivially $a \leqslant \Delta b$.

- If there is no $c \in(0,1)_{\mathbb{Q}}$ such that $b \leqslant c^{\boldsymbol{A}}$, since $\boldsymbol{A}$ is linearly ordered we have that $c^{\boldsymbol{A}} \leqslant b$ for all $c \in(0,1)_{\mathbb{Q}}$. Then, by $\mathcal{Q}_{\Delta}, b=1$ and trivially $a \leqslant \Delta b=1$.

From this result, one can build a complete embedding from any chain in $\mathbb{P}^{\infty}$ into $[0,1]_{\Pi}$ in a natural way. It is just necessary to ensure that the constants are sent to their correspondent value in $[0,1]$.

Lemma 3.9. Let $\boldsymbol{A}$ be a $\Pi^{\infty}$-algebra linearly ordered. Then $\boldsymbol{A}$ can be embedded in the canonical standard product algebra $[0,1]_{\Pi}$ by a complete embedding.

Proof. The details of the proof, being rather technical and not necessary for the comprehension of the general result, can be found in the Annex.

We can finally state the completeness result as a corollary from the previous Lemmas. 
Theorem 3.10 (Strong Standard Completeness). For any $\Gamma \cup\{\varphi\} \subseteq F m_{\Pi^{\infty}}$, the following are equivalent:

(1) $\Gamma \vdash \Pi_{\infty} \varphi$;

(2) $\Gamma \models \mathbb{P} \infty$;

(3) $\Gamma \models_{C \mathbb{P}^{\infty}} \varphi$, where $C \mathbb{P}^{\infty}$ denotes the class of chains in $\mathbb{P}^{\infty}$;

(4) $\Gamma \models[0,1]_{\Pi} \varphi$.

Proof. (1) $\Rightarrow(4)$ is the soundness of the axioms and rules, and it is a matter of routine checking. On the other hand, $(4) \Rightarrow(3)$ follows directly from Lemma 3.9 , while $(3) \Rightarrow(2)$ is the strong completeness of $\Pi^{\infty}$ with respect to the linearly ordered $\Pi^{\infty}$-algebras, and this is Corollary 3.6. Finally, $(2) \Rightarrow(1)$ is the completeness result stated in condition (1), that is a consequence of $\Pi^{\infty}$ being a Rasiowa-implicative logic.

A remarkable fact that arises from this strong completeness result is the very natural behaviour of the constants over any $\Pi^{\infty}$-algebra.

Lemma 3.11. Let $\boldsymbol{A} \in \mathbb{P}^{\infty}$ and $a, b \in \boldsymbol{A}$. If $a<b$ then there is $c \in(0,1)_{\mathbb{Q}}$ such that $c^{\boldsymbol{A}} \nless a$ and $b \not c^{A}$.

Proof. Using the fact that the rational numbers are dense in the real interval $[0,1]$ it is clear that for any $x, y \in[0,1]$ we have that if $x \leqslant y$ and $\left(c^{\boldsymbol{A}} \rightarrow x\right) \vee\left(y \rightarrow c^{\boldsymbol{A}}\right)=1$ for each $c \in(0,1)_{\mathbb{Q}}$ then $y \leqslant x$ (i.e., $y=x$ ). This can be written as, for any $\varphi, \psi \in F m$,

$$
\left.\{\varphi \rightarrow \psi\} \cup\{(\bar{c} \rightarrow \varphi) \vee(\psi \rightarrow \bar{c})\}_{c \in(0,1)_{\mathbb{Q}}}\right|_{[0,1]_{\Pi}} \psi \rightarrow \varphi
$$

By the strong completeness, it follows that

$$
\{\varphi \rightarrow \psi\} \cup\{(\bar{c} \rightarrow \varphi) \vee(\psi \rightarrow \bar{c})\}_{c \in(0,1)_{\mathbb{Q}}} \vdash_{\Pi^{\infty}} \psi \rightarrow \varphi,
$$

and thus, by completeness with respect to the whole class of algebras, we have that, in an arbitrary $\boldsymbol{A}$, if $a<b \in \boldsymbol{A}$ there must exist $c_{0} \in(0,1)_{\mathbb{Q}}$ for which $\left(c_{0}^{\boldsymbol{A}} \rightarrow a\right) \vee\left(b \rightarrow c_{0}^{\boldsymbol{A}}\right)<1$ (otherwise, $b=a$ ). Then, in particular, $c_{0}^{\boldsymbol{A}} \rightarrow a<1$ and $b \rightarrow c_{0}^{\boldsymbol{A}}<1$, which means, by the definition of the order in $\boldsymbol{A}$, that $c_{0}^{\boldsymbol{A}} \not a$ and $b \not c_{0}^{\boldsymbol{A}}$.

Corollary 3.12. If $\boldsymbol{A} \in \mathbb{P}^{\infty}$ is linearly ordered, then for each $a<b \in \boldsymbol{A}$ there exists $c \in(0,1)_{\mathbb{Q}}$ such that $a<c^{\boldsymbol{A}}<b$.

\section{Minimal Local and global modal logics Over $\Pi^{\infty}$}

In this section we will focus on the study of the local and global modal logics associated with the class of product algebras with $\Delta$ and rational constants. It is important to remark that we will consider the modal logics as closure operators, not just as a closed set of formulas (theorems), studying this way a richer problem whose solutions have implicit the other possible definition of the logic. The main result presented is the definition of a axiomatic systems that are (respectively) strongly complete with respect to the local and global modal logics that arise from the class of crisp Kripke models evaluated over Product algebras, and in particular, with respect to Kripke models evaluated over the canonical standard product algebra.

We will begin by defining the modal logic in semantic terms. First, we let the modal language be the expansion of the non-modal language for product logic with rational constant symbols and $\Delta$ operator with two unary operators, $\square$ and $\diamond$. From now on, unless specified otherwise $\boldsymbol{F} \boldsymbol{m}$ will denote the the algebra of formulas built from a countable set of variables $\mathcal{V}$ and rational constants $\{\bar{c}\}_{c \in[0,1]_{\mathbb{Q}}}$ using the connectives of the modal language. 
The notion of Kripke frame is the usual one: a pair $\langle W, R\rangle$ where $W$ is a non-empty set of worlds and $R \subseteq W \times W$ is an accessibility relation ${ }^{5}$. For simplicity on the notation, we will write $R v w$ whenever $\langle v, w\rangle \in R$.

Definition 4.1. Let $\mathbf{A} \in \mathbb{P}^{\infty}$. An A-Kripke model $\mathfrak{M}=\langle W, R, e\rangle$ is a Kripke frame $\langle W, R\rangle$ endowed with an evaluation of propositional variables on $\mathbf{A}$ for each world, $e: W \times \mathcal{V} \rightarrow \mathbf{A}$. The map $e$ is extended to non-modal formulas by their corresponding operations in $\mathbf{A}$, i.e., fulfilling $e(v, \varphi \& \psi)=e(v, \varphi) \odot e(v, \psi), e(v, \varphi \rightarrow \psi)=e(v, \varphi) \rightarrow e(v, \psi), e(v, \Delta \varphi)=\Delta e(v, \varphi)$ and $e(v, \bar{c})=$ $c^{\mathbf{A}}$, and to modal formulas by the following expressions:

$$
e(v, \square \varphi)=\inf \{e(w, \varphi): \operatorname{Rvw}\} \quad e(v, \diamond \varphi)=\sup \{e(w, \varphi): \operatorname{Rvw}\}
$$

whenever these values are defined, and are left undefined otherwise.

A model $\mathfrak{M}$ is called safe whenever the evaluation of $\square \varphi$ and $\diamond \varphi$ is defined over any world for any $\varphi \in F m$. For the sake of simplicity, from now on we will only consider safe models, and we will denote the class of safe models over the algebras in $\mathbb{P}^{\infty}$ is denoted by PK, and its elements will be called Product Kripke models.

Over the previous class of models PK, different notions of truth arise. Given $\varphi \in F m, \mathfrak{M} \in \mathrm{PK}$ and $w \in W$, we write $(\mathfrak{M}, w) \models \varphi$ whenever $e(w, \varphi)=1$, and $\mathfrak{M} \models \varphi$ whenever $(\mathfrak{M}, w) \models \varphi$ for all $w \in W$. Then, as usual, two logical consequences can be defined, a local and a global one. For an arbitrary set of formulas $\Gamma \cup\{\varphi\} \subseteq F m$, we define them by:

- $\Gamma \models_{\mathrm{PK}}^{l} \varphi$ if for any $\mathfrak{M} \in \mathrm{PK}$ and $w \in W,(\mathfrak{M}, w) \models \Gamma$ implies that $(\mathfrak{M}, w) \models \varphi$.

- $\Gamma \models_{\mathrm{PK}}^{g} \varphi$ if for any $\mathfrak{M} \in \mathrm{PK}, \mathfrak{M}=\Gamma$ implies that $\mathfrak{M}=\varphi$.

Notice that the local deduction is strictly weaker than the global one, since $\Gamma \models_{\text {PK }}^{l} \varphi$ implies that $\Gamma \models_{\mathrm{PK}}^{g} \varphi$, but not the contrary. It is also remarkable that the set of theorems (i.e., deductions from the empty set) under the local and the global deduction coincide.

The main goal is to provide a complete axiomatization for these logics. We will focus on the proof of completeness of the axiomatization of the local consequence, since the results needed in the case of the global consequence can be easily obtained from the ones of the local one. The proposed axiomatization for the local consequence $\models_{\mathrm{PK}}^{l}$ is the following.

Definition 4.2. $\mathbf{K} \Pi^{\infty}$ is the axiomatic system on the modal language given by the extension of $\Pi^{\infty}$ by the following axioms and rules:

$(\mathrm{K}) \square(\varphi \rightarrow \psi) \rightarrow(\square \varphi \rightarrow \square \psi)$

$\left(\mathrm{A}_{\square} 1\right) \square(\bar{c} \rightarrow \varphi) \leftrightarrow(\bar{c} \rightarrow \square \varphi)$

$\left(\mathrm{A}_{\diamond} 1\right) \square(\varphi \rightarrow \bar{c}) \leftrightarrow(\diamond \varphi \rightarrow \bar{c})$

$\left(\mathrm{A}_{\square} 2\right) \Delta \square \varphi \leftrightarrow \square \Delta \varphi$

$\left(\mathrm{N}_{\square}\right)$ From $\vdash \varphi$ derive $\vdash \square \varphi$.

As usual, for an arbitrary set of formulas $\Gamma \cup\{\varphi\} \subseteq F m$, we will write $\Gamma \vdash_{\mathbf{K} \Pi \infty} \varphi$ whenever there is a proof (in the sense of Definition 3.3) of $\varphi$ from elements in $\Gamma$ (clearly, we are assuming the deductions are closed under uniform substitution). ${ }^{6}$

The axiomatization corresponding to the global consequence $\models_{\mathrm{PK}}^{g}$ will be denoted by $\mathbf{G K} \Pi^{\infty}$. Its definition is as follows.

Definition 4.3. GK $\Pi^{\infty}$ is the axiomatic system resulting from $\mathbf{K} \Pi^{\infty}$ by just replacing the local necessitation rule $\left(\mathrm{N}_{\square}\right)$ by the more general rule:

\footnotetext{
${ }^{5}$ It is not in the scope of this paper to deal with fuzzy accesibility relations, where $R: W \times W \rightarrow \mathbf{A}$ for some algebra $\mathbf{A} \in \mathbb{P}^{\infty}$.

${ }^{6}$ Note we could restrict ourselves to study the finitary modal logic $\left(\Gamma \vdash_{\mathbf{K} \Pi \infty}^{f} \varphi\right.$ whenever there is a proof, also in the sense of Definition 3.3, of $\varphi$ from a finite subset of $\Gamma$ ), but as we will see, no extra assumptions are needed to work directly with the infinitary case (since the infinitary flavour is treated already at the non-modal level).
} 


\section{$\left(N_{\square}^{g}\right)$ From $\varphi$ derive $\square \varphi$}

The corresponding notion of proof will be denoted by $\vdash_{\mathbf{G K} \Pi \infty}$.

Note that the rule $\left(N_{\square}^{g}\right)$ is to be applied to any formula, not only to theorems as with the rule $\left(\mathrm{N}_{\square}\right)$.

As it happened at the semantical level, $\vdash_{\mathbf{K} \Pi^{\infty}}$ is weaker than $\vdash_{\mathbf{G K} \Pi^{\infty}}$. It is also clear that the theorems of both logics coincide, and we will refer to this set by $T h_{\mathbf{K} \Pi \infty}$.

The soundness of the previous axiomatizations with respect to their semantic counterpart is easy to check and we omit the proof. To show that $\mathbf{K} \Pi^{\infty}$ indeed axiomatizes the local consequence $\left.\right|_{\text {PK }} ^{l}$ we have to prove that, for any set of formulas $\Gamma \cup\{\varphi\} \subseteq F m$, if $\Gamma \nvdash_{\mathbf{K} \Pi{ }^{\infty} \varphi} \varphi$ there exist a model $\mathfrak{M}=\langle W, R, e\rangle \in \mathrm{PK}$ and a world $w \in W$ for which $(\mathfrak{M}, w) \models \Gamma$ but $(\mathfrak{M}, w) \not \models \varphi$. This will be done through the usual canonical model construction. But first, we will present some results about $\mathbf{K} \Pi^{\infty}$ that will be useful for this task.

Since the only modal deduction rule of $\mathbf{K} \Pi^{\infty}$ applies just to theorems, it is clear that the $\Delta$-Deduction Theorem of $\Pi^{\infty}$ (see Section 2) keeps holding, i.e.,

$$
\Gamma \cup\{\alpha\} \vdash_{\mathbf{K} \Pi \infty} \varphi \text { iff } \Gamma \vdash_{\mathbf{K} \Pi \infty} \Delta \alpha \rightarrow \varphi .
$$

However, the fact that $\mathbf{K} \Pi^{\infty}$ is not finitary imposes a detour in the completeness proof. Indeed, if the logic were finitary, the Deduction Theorem would allow to treat the logic as just a set of theorems, and then the proof of correctness of the canonical model could be more directly done simply using the rule $\left(\mathrm{N}_{\square}\right)$. In this more general context, it is necessary to show that the deductions in $\mathbf{K} \Pi^{\infty}$ are closed under the $\square$ operator.

Lemma 4.4. Let $\Gamma \cup\{\varphi\} \subseteq F m$. $\Gamma \vdash_{\mathbf{K} \Pi \infty} \varphi$ implies that $\square \Gamma \vdash_{\mathbf{K} \Pi \infty} \square \varphi$, where $\square \Gamma$ stands for $\{\square \gamma: \gamma \in \Gamma\}$.

Proof. Applying induction on the depth ${ }^{7}$ of the proof of $\varphi$ from $\Gamma$, we consider the possible last step in that proof:

- If $\varphi$ is a theorem, then by $\left(\mathrm{N}_{\square}\right), \square \varphi$ is a theorem too.

- If $\varphi$ was obtained by $(M P)$, then $\Gamma \vdash_{\mathbf{K} \Pi \infty} \psi$ and $\Gamma \vdash_{\mathbf{K} \Pi \infty} \psi \rightarrow \varphi$. Applying the induction hypothesis, axiom (K) and the $(M P)$ rule, it follows that $\square \Gamma \vdash_{\mathbf{K} \Pi \infty} \square \varphi$.

- If $\varphi$ is $\Delta \psi$ and it was obtained by $\left(G_{\Delta}\right)$, then $\Gamma \vdash_{\mathbf{K} \Pi^{\infty}} \psi$. Applying the induction hypothesis, the rule $\left(G_{\Delta}\right)$ and axiom $\left(\mathrm{A}_{\square} 2\right)$, we get that $\square \Gamma \vdash_{\mathbf{K} \Pi \infty} \square \Delta \psi$.

- If $\varphi$ was obtained by the rule $\left(R_{\Delta}\right)$, then $\Gamma \vdash_{\mathbf{K} \Pi \infty} \bar{c} \rightarrow \varphi$ for all $c \in(0,1)_{\mathbb{Q}}$. By the induction hypothesis we get $\square \Gamma \vdash_{\mathbf{K} \Pi \infty} \square(\bar{c} \rightarrow \varphi)$ for all $c \in(0,1)_{\mathbb{Q}}$, and thus, by axiom $\left(\mathrm{A}_{\square} 1\right)$ it

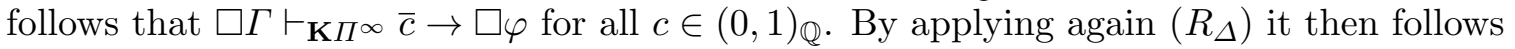
that $\square \Gamma \vdash_{\mathbf{K} \Pi \infty} \square \varphi$.

- If $\varphi$ is $\neg \psi$ and it was obtained by the rule $\left(R_{\rightarrow}\right)$, then $\Gamma \vdash_{\mathbf{K} \Pi \infty} \psi \rightarrow \bar{c}$ for all $c \in(0,1)_{\mathbb{Q}}$. As before, by applying the induction hypothesis, $\left(\mathrm{A}_{\diamond} 1\right),\left(R_{\rightarrow}\right)$ and again $(\mathrm{A} \diamond 1)$, we conclude that $\square \Gamma \vdash_{\mathbf{K} \Pi \infty} \square \neg \psi$.

One of the usual methods to prove completeness in modal logics is by defining a suitable translation of modal formulas into non-modal ones over an extended set of variables, and then translating proofs in the modal logic into proofs from an extended set of premises (possibly infinite) in the underlying non-modal logic. Actually, this is the reason behind the necessity of having strong completeness at the non-modal level. In our case, we can still proceed in this way thanks to the fact that $\Pi^{\infty}$ is strongly complete and any use of $\left(\mathrm{N}_{\square}\right)$ rule in a proof in $\mathbf{K} \Pi^{\infty}$ produces a theorem of the logic.

Formally, we denote by $\mathcal{V}^{\star}$ the extended set of variables $\mathcal{V} \cup\left\{\varphi_{\square}, \varphi_{\diamond}\right\}_{\varphi \in F m}$, and by $F m^{\star}$ the set of formulas in the language of $\Pi_{\Delta}^{c}$ (without modal operators) over the extended set of variables $\mathcal{V}^{\star}$.

\footnotetext{
${ }^{7}$ Recall that proofs are defined in Definition 3.3 as trees of finite depth.
} 
Definition 4.5. We inductively define a one-to-one translation to non-modal formulas $\star: F m \rightarrow$ $F m^{\star}$ by

$$
\begin{aligned}
\bar{c}^{\star}:=\bar{c}, \quad x^{\star}:=x \text { for } x \in \mathcal{V} & (\Delta \varphi)^{\star}:=\Delta \varphi^{\star} \\
(\varphi \& \psi)^{\star}:=\varphi^{\star} \& \psi^{\star} & (\square \varphi)^{\star}:=\varphi \square \\
(\varphi \rightarrow \psi)^{\star}:=\varphi^{\star} \rightarrow \psi^{\star} & (\diamond \varphi)^{\star}:=\varphi \diamond .
\end{aligned}
$$

Note that the modal axioms are simply translated to the following schemes:

$$
\begin{array}{rlrl}
(\mathrm{K})^{\star}:(\varphi \rightarrow \psi)_{\square} & \rightarrow\left(\varphi_{\square} \rightarrow \psi_{\square}\right) & & \left(\mathrm{A}_{\diamond} 1\right)^{\star}:(\varphi \rightarrow \bar{c})_{\square} \leftrightarrow\left(\varphi_{\diamond} \rightarrow \bar{c}\right) \\
\left(\mathrm{A}_{\square} 1\right)^{\star}:(\bar{c} \rightarrow \varphi)_{\square} \leftrightarrow\left(\bar{c} \rightarrow \varphi_{\square}\right) & & \left(\mathrm{A}_{\square} 2\right)^{\star}: \Delta \varphi_{\square} \leftrightarrow(\Delta \varphi)_{\square}
\end{array}
$$

This translation can be used to obtain an equivalence between deductions in the local modal logic and deductions in the non-modal product logic. It is immediate, since all the propositional rules are shared and all the formulas deduced in $\mathbf{K} \Pi^{\infty}$ by the $\left(\mathrm{N}_{\square}\right)$ rule belong to $T h_{\mathbf{K} \Pi^{\infty}}$.

Lemma 4.6. For any set of formulas $\Gamma \cup\{\varphi\} \subseteq F m, \Gamma \vdash_{\mathbf{K} \Pi \infty} \varphi$ if and only if $\Gamma^{\star} \cup\left(T h_{\mathbf{K} \Pi \infty}\right)^{\star} \vdash_{\Pi^{\infty}}$ $\varphi^{\star}$.

To prove strong standard completeness of $\mathbf{K} \Pi^{\infty}$ with respect to the local deduction $\models_{\text {PK }}^{l}$ we will

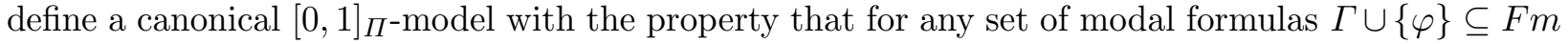
such that $\Gamma \forall_{\mathbf{K} \Pi \infty} \varphi$, there is a world in the model which assigns the value 1 to all $\gamma \in \Gamma$ but a value strictly smaller than 1 to $\varphi$.

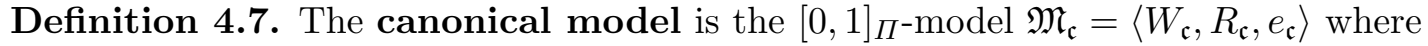

- $W_{\mathfrak{c}}:=\left\{h \in \operatorname{Hom}\left(\mathbf{F m}^{\star},[0,1]_{\Pi}\right): h\left(\left[\left(T h_{\mathbf{K} \Pi \infty}\right)^{\star}\right]\right) \subseteq\{1\}\right\}$;

- $R_{\mathfrak{c}} v w$ if for any $\psi \in F m$ such that $v\left(\psi_{\square}\right)=1$ it holds that $w\left(\psi^{\star}\right)=1$;

- $e_{\mathfrak{c}}(w, p):=w(p)$, for every $p \in \mathcal{V}$.

One may wonder about the above definition of $R_{\mathfrak{c}}$ as it is only depends on the $\square$ modality and on formulas evaluated to 1 . However as next lemmas show, this suffices to prove the truth lemma for this canonical model.

Lemma 4.8. $R_{\mathfrak{c}} v w$ if and only if for any $\psi \in F m$, both inequalities hold:

- $v\left(\psi_{\square} \leqslant w\left(\psi^{\star}\right)\right.$;

- $v\left(\psi_{\diamond}\right) \geqslant w\left(\psi^{\star}\right)$.

Proof. To prove the non-direct case, assume $v\left(\psi_{\square}\right)>w\left(\psi^{\star}\right)$ for some $\psi \in F m$. Then there is $c \in(0,1)_{\mathbb{Q}}$ such that $w\left(\psi^{\star}\right)<c<v\left(\psi_{\square}\right)$, and so, $1=c \Rightarrow_{\Pi} v\left(\psi_{\square}\right)=v\left(\bar{c} \rightarrow \psi_{\square}\right)$. Axiom $\left(\mathrm{A}_{\square} 1\right)^{\star}$ leads to $1=v\left((\bar{c} \rightarrow \psi)_{\square}\right)$. However, $w\left((\bar{c} \rightarrow \psi)^{\star}\right)=c \Rightarrow_{\Pi} w\left(\psi^{\star}\right)<1$, and so it does not hold that $R_{\mathfrak{c}} v w$. The other proof, that $v\left(\psi_{\diamond}\right)<w\left(\psi^{\star}\right)$ leads to that $v$ is not related with $w$, is analogous using $\left(\mathrm{A}_{\diamond} 1\right)^{\star}$.

In the way of proving the Truth Lemma for the canonical model, we first show a crucial result.

Lemma 4.9. Let $v \in W_{\mathfrak{c}}$ and $\varphi \in F m$ such that $w\left(\varphi^{\star}\right)=1$ for all $w \in W_{\mathfrak{c}}$ such that $R_{\mathfrak{c}} v w$. Then $v(\varphi \square)=1$ too.

Proof. Let $w \in W_{\mathfrak{c}}$. Then, by definition, $R_{\mathfrak{c}} v w$ if and only if $w\left(\left[\left(T h_{\mathbf{K} \Pi^{\infty}}\right)^{\star}\right]\right) \subseteq\{1\}$ and $w\left(\psi^{\star}\right)=1$ for all $\psi \in F m$ such that $v\left(\psi_{\square}\right)=1$. In other words, for any $w \in \operatorname{Hom}\left(\mathbf{F m}^{*},[0,1]_{\Pi}\right), R_{\mathfrak{c}} v w$ if and only if $w\left(\left[\left(T h_{\mathbf{K} \Pi^{\infty}} \cup T\right)^{\star}\right]\right) \subseteq\{1\}$, where $T=\left\{\psi \in F m: v\left(\psi_{\square}\right)=1\right\}$.

Therefore, the hypothesis of the lemma amounts to assume $\left(T h_{\mathbf{K} \Pi \infty} \cup T\right)^{\star} \models_{[0,1]_{\Pi}} \varphi^{\star}$. By strong

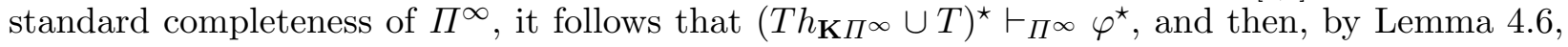
$T \vdash_{\mathbf{K} \Pi \infty} \varphi$ as well.

Now Lemma 4.4 can be applied, obtaining $\square T \vdash_{\mathbf{K} \Pi \infty} \square \varphi$. By the same reasoning in the opposite sense (Lemma 4.6 and then applying strong standard completeness), it follows that $\left(T h_{\mathbf{K} \Pi \infty \cup}\right.$ 


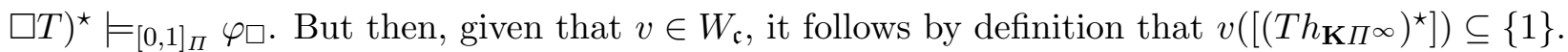
On the other hand, for each $\psi \in T$ it holds that $v\left(\psi_{\square}\right)=1$, hence $v\left(\left[(\square T)^{\star}\right]\right) \subseteq\{1\}$. Therefore, since the whole set of premises is evaluated to 1 , it also holds that $v(\varphi \square)=1$, which concludes the proof.

Using the density of the rational numbers within the reals, we get the Truth Lemma as a consequence of the previous result.

Lemma 4.10 (Truth Lemma). For any $\varphi \in F m$ and any $v \in W_{\mathfrak{c}}$ it holds that

$$
e(v, \varphi)=v\left(\varphi^{\star}\right) \text {. }
$$

Proof. This can be proven by induction on the structure of the formulas, and the only cases that are worth to be detailed are the modal ones.

To show that $v\left(\varphi_{\square}\right)=\inf \left\{w\left(\varphi^{\star}\right): R_{\mathfrak{c}} v w\right\}$, first notice that by Lemma 4.8, $v\left(\varphi_{\square}\right) \leqslant w\left(\varphi^{\star}\right)$ for any $w$ such that $R_{\mathfrak{c}} v w$, so $v\left(\varphi_{\square}\right) \leqslant \inf \left\{w\left(\varphi^{\star}\right): R_{\mathfrak{c}} v w\right\}$. To prove the converse inequality, by way of a contradiction assume that $v\left(\varphi_{\square}\right)<\inf \left\{w\left(\varphi^{\star}\right): R_{\mathfrak{c}} v w\right\}$. Then there is $c \in(0,1)_{\mathbb{Q}}$ such that $v\left(\varphi_{\square}\right)<c<w\left(\varphi^{\star}\right)$ for any $w$ such that $R_{\mathfrak{c}} v w$. This implies that $w\left((\bar{c} \rightarrow \varphi)^{\star}\right)=1$ for any $w$ such that $R_{\mathfrak{c}} v w$, and by Lemma 4.9 we have that $v\left((\bar{c} \rightarrow \varphi)_{\square}\right)=1$ as well. However, by axiom $\left(\mathrm{A}_{\square} 1\right)^{\star}$, $v\left((\bar{c} \rightarrow \varphi)_{\square}\right)=v\left(\bar{c} \rightarrow \varphi_{\square}\right)$, and hence $c \rightarrow v\left(\varphi_{\square}\right)=1$ too, which contradicts the fact that $v\left(\varphi_{\square}\right)<c$.

To show that $v\left(\varphi_{\diamond}\right)=\sup \left\{w\left(\varphi^{\star}\right): R_{\mathfrak{c}} v w\right\}$, the proof is analogous using axiom $\left(\mathrm{A}_{\diamond} 1\right)^{\star}$ instead of $\left(\mathrm{A}_{\square} 1\right)^{\star}$, when needed.

Now, completeness of $\mathbf{K} \Pi^{\infty}$ with respect to the local semantics is a quite direct corollary.

Theorem 4.11 (Completeness of $\mathbf{K} \Pi^{\infty}$ ). For any set of modal formulas $\Gamma \cup\{\varphi\}$ the following are equivalent:

(1) $\Gamma \vdash_{\mathbf{K} \Pi \infty \varphi} \varphi$;

(2) $\Gamma \models_{[0,1]_{\Pi} \mathrm{K}}^{l} \varphi$, where $[0,1]_{\Pi} \mathrm{K}$ denotes the class of $[0,1]_{\Pi}$-Kripke models; ${ }^{8}$

(3) $\Gamma=_{\mathcal{C P K}}^{l} \varphi$, where $\mathcal{C P K}$ denotes the class of safe $\mathbf{A}$-Kripke models such that $\mathbf{A} \in \mathbb{P}^{\infty}$ is a chain:

(4) $\Gamma \models{ }_{\mathrm{PK}}^{l} \varphi$.

Proof. Observe that (4) implies (3), and that (3) implies (2). Soundness is simple to check in general for the $\left.\right|_{\text {PK }} ^{l}$ case, so (1) implies (4). Therefore it remains to prove (2) implies (1), using the canonical model, and the rest follows directly.

Assume $\Gamma \forall_{\mathbf{K} \Pi^{\infty}} \varphi$. By Lemma 4.6, this happens if and only if $\Gamma^{\star} \cup\left(T h_{\mathbf{K} \Pi^{\infty}}\right)^{\star} \nvdash_{\Pi^{\infty}} \varphi^{\star}$. By strong completeness of $\Pi^{\infty}$, there is an homomorphism $v$ from $F m^{\star}$ into the canonical standard product algebra $[0,1]_{\Pi}$ such that $v\left(\left[\left(T h_{\mathbf{K} \Pi^{\infty}}\right)^{\star}\right]\right) \subseteq\{1\}$ and $v\left(\left[\Gamma^{\star}\right]\right) \subseteq\{1\}$ but $v\left(\varphi^{\star}\right)<1$. Hence, $v \in W_{\mathfrak{c}}$ and by the Truth Lemma 4.10,e $e(v,[\Gamma])=v\left(\left[\Gamma^{\star}\right]\right) \subseteq\{1\}$ and $e(v, \varphi)=v\left(\varphi^{\star}\right)<1$, so $\left.\mathfrak{M}_{\mathfrak{c}}\right|_{v} \Gamma$ and $\mathfrak{M}_{\mathfrak{c}} \mid \nvdash_{v} \varphi$.

Theorem 4.12 (Completeness GK $\Pi^{\infty}$ ). For any set of (modal) formulas $\Gamma \cup\{\varphi\}$ the following are equivalent:

(1) $\Gamma \vdash_{\mathbf{G K} \Pi \infty \varphi} \varphi$

(2) $\Gamma \models_{[0,1]_{\Pi} \mathrm{K}}^{g} \varphi$;

(3) $\Gamma \models \stackrel{g}{g} \models_{g}^{g} \varphi$;

(4) $\Gamma \models{ }_{\mathrm{PK}}^{g} \varphi$.

Proof. (Sketch) The proof of completeness of the logic GK $\Pi^{\infty}$ with respect to the global consequence relation $={ }_{\mathrm{PK}}^{g}$ can be proved in a similar way to the local one. The main difference is defining a slightly different canonical model $\mathfrak{M}_{\mathfrak{c}}{ }^{\Gamma}$ for each set of formulas $\Gamma \cup\{\varphi\} \subseteq F m$ such that $\Gamma \forall_{\mathbf{G K} \Pi \infty} \varphi$. For each

\footnotetext{
${ }^{8}$ Observe $[0,1]_{\Pi}$ is a complete algebra, and so all the models defined over it are safe.
} 
of such sets, the worlds of $\mathfrak{M}_{\mathfrak{c}}{ }^{\Gamma}$ are defined as the homomorphisms in $\operatorname{Hom}\left(\mathbf{F m}^{\star},[0,1]_{\Pi}\right)$ that satisfy

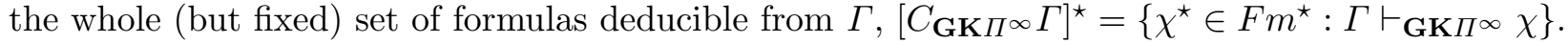
To prove that the model so defined enjoys the Truth Lemma, the simplest way is to resort to the results concerning the local logic, already proven. In particular, we can prove the result corresponding to Lemma 4.9 in this new context using the same methods (and with that we mean resorting to the local logic $\mathbf{K} \Pi^{\infty}$ in the proof). Finally, the result analogous to Lemma 4.6 that is now needed turns to be no more than an obvious observation: $\Gamma \forall_{\mathbf{G K} \Pi^{\infty}} \varphi$ if and only if $\left[C_{\mathbf{G K} \Pi^{\infty}} \Gamma\right]^{\star} \nvdash_{\Pi^{\infty}} \varphi^{\star}$ This points out an homomorphism $h$ within the standard product algebra, $[0,1]_{\Pi}$, such that

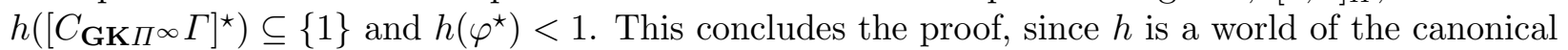
model $\mathfrak{M}_{\mathfrak{c}}{ }^{\Gamma}$ (which is a global model for $\Gamma$ by definition).

\section{Algebraic semantics for the Product modal LOGics}

In this section we will study the algebraic semantics of the modal systems $\mathbf{K} \Pi^{\infty}$ and $\mathbf{G K} \Pi^{\infty}$. We will begin classifying $\mathbf{K} \Pi^{\infty}$ and $\mathbf{G K} \Pi^{\infty}$ into the Leibniz Hierarchy and obtain their algebraic classes, and then proceed to study the classes of matrices over these algebras corresponding to each logic, providing completeness results more specific than the general one (of each logic with respect to its reduced models). We will finish by studying the relation between the Kripke and the algebraic semantics.

\subsection{Algebraic semantics for $\mathrm{K} \Pi^{\infty}$ and $\mathrm{GK} \Pi^{\infty}$.}

For the algebraic counterpart of $\mathbf{K} \Pi^{\infty}$ and $\mathbf{G K} \Pi^{\infty}$ it is natural to consider the class of algebras obtained trough the expansion of the product algebras in $\mathbb{P}^{\infty}$ by modal operators that satisfy the equations induced by the theorems in $\mathbf{K} \Pi^{\infty}$.

Definition 5.1. An algebra $\boldsymbol{A}=\left\langle A, \odot, \rightarrow, \Delta, \square, \diamond,\left\{c^{\boldsymbol{A}}\right\}_{c \in[0,1]_{\mathbb{Q}}}\right\rangle$ is a modal product algebra with (rational) constants and $\Delta$, or a $\mathbf{K} \Pi^{\infty}$-agebra, whenever $\left\langle A, \odot, \rightarrow, \Delta,\left\{c^{\boldsymbol{A}}\right\}_{c \in[0,1]_{\mathbb{Q}}}\right\rangle \in \mathbb{P}^{\infty}$ and the following equations hold:

$\left(\mathrm{E}_{\mathrm{K}}\right) \square(x \rightarrow y) \rightarrow(\square x \rightarrow \square y) \approx 1^{A} ;$

$\left(\mathrm{E}_{\left.\mathrm{A}_{\square}\right)}\right) \square\left(c^{\boldsymbol{A}} \rightarrow x\right) \approx c^{\boldsymbol{A}} \rightarrow \square x$, for every $c \in[0,1]_{\mathbb{Q}} ;$

$\left(\mathrm{E}_{\mathrm{A} \diamond 1}\right) \square\left(x \rightarrow c^{\boldsymbol{A}}\right) \approx \diamond x \rightarrow c^{\boldsymbol{A}}$, for every $c \in[0,1]_{\mathbb{Q}} ;$

$\left(\mathrm{E}_{\mathrm{A}_{\square} 2}\right) \square \Delta x \approx \Delta \square x ;$

$\left(\mathrm{E}_{\mathrm{N}_{\square}}\right) \square 1^{A} \approx 1^{A}$.

We will denote by $\mathbb{K} \mathbb{P}^{\infty}$ the class of $\mathbf{K} \Pi^{\infty}$-agebra, and for each $\boldsymbol{A} \in \mathbb{K} \mathbb{P}^{\infty}, \overline{\boldsymbol{A}}$ will denote its non-modal reduct.

A modal evaluation $e$ over a modal product algebra $\boldsymbol{A}$ is an homomorphism from the algebra of modal formulae (with $\Delta$ and rational constants) into $\boldsymbol{A}$.

From the general definition of a filter, we have that the deductive filters of the non-modal logic $\Pi^{\infty}$ over a product algebra $\boldsymbol{A} \in \mathbb{P}^{\infty}$ are given by subsets $F \subseteq A$ such that

$-1^{A} \in F$,

- If $x \in F$ and $x \rightarrow y \in F$ then $y \in F$,

- If $x \in F$ then $\Delta x \in F$,

- If $c^{\boldsymbol{A}} \rightarrow x \in F$ for all $c \in(0,1)_{\mathbb{Q}}$ then $x \in F$,

- If $x \rightarrow c^{\boldsymbol{A}} \in F$ for all $c \in(0,1)_{\mathbb{Q}}$ then $\neg x \in F$.

The filters of $\mathbf{K} \Pi^{\infty}$ are those of $\Pi^{\infty}$ that are also closed under $N_{\square}$ (i.e., $\square 1^{\boldsymbol{A}} \in F$ ), and those of GK $\Pi^{\infty}$ will be those of of $\Pi^{\infty}$ closed under $N_{\square}^{g}$ (i.e., if $x \in F$ then $\square x \in F$ ).

In order to simplify the notation in the characterization of the filters of the modal logics, we shall introduce a natural concept. We say that a subset $X$ of an algebra $\boldsymbol{A} \in \mathbb{K} \mathbb{P}^{\infty}$ is open whenever for any $x \in X$, it holds that $\square x \in X$ as well. 
Then, we can characterize more clearly the filters of $\mathbf{K} \Pi^{\infty}$ and $\mathbf{G K} \Pi^{\infty}$ over a modal product algebra $\boldsymbol{A} \in \mathbb{K} \mathbb{P}^{\infty}$.

Lemma 5.2. Given $\boldsymbol{A} \in \mathbb{K} \mathbb{P}^{\infty}$, and $F \subseteq A$

- $F$ is a filter of $\mathbf{K} \Pi^{\infty}$ over $\boldsymbol{A}$ if and only if $F \in \mathcal{F} i_{\Pi \infty} \overline{\boldsymbol{A}}$

- $F$ is a filter of $\mathbf{G K} \Pi^{\infty}$ over $\boldsymbol{A}$ if and only if $F \in \mathcal{F} i_{\Pi^{\infty}} \overline{\boldsymbol{A}}$ and $F$ is an open set.

It is routine to prove that $\left\{1^{\boldsymbol{A}}\right\}$ is a filter of both of $\mathbf{K} \Pi^{\infty}$ and of $\mathbf{G K} \Pi^{\infty}$ logics over all $\boldsymbol{A} \in \mathbb{K P}^{\infty}$.

It turns out that the classification of the global modal logic $\mathbf{G K} \Pi^{\infty}$ in the Leibniz Hierachy is now quite immediate, just by checking the definition.

Lemma 5.3. GK $\Pi^{\infty}$ is algebraizable with algebraic semantics $\mathbb{K} \mathbb{P}^{\infty}$, with equivalence formulas $\boldsymbol{\Delta}(x, y):=\{x \leftrightarrow y\}$ and with defining equations $E(x)=\{x \approx 1\}$.

From this it follows that $A l g^{*} \mathbf{G K} \Pi^{\infty}=\mathbb{K} \mathbb{P}^{\infty}$ and thus, its reduced models are of the form $\left\langle\boldsymbol{A},\left\{1^{\boldsymbol{A}}\right\}\right\rangle$ for $\boldsymbol{A} \in \mathbb{K}^{\infty}$. That is to say, we have the following result.

Theorem 5.4 (Algebraic Completeness of GK $\Pi^{\infty}$ ). For any set of modal formulas $\Gamma \cup\{\varphi\}$

$$
\Gamma \vdash_{\mathbf{G K} \Pi \infty} \varphi \text { iff } \Gamma \models_{\mathbb{K} \mathbb{P} \infty} \varphi \text {. }
$$

This fact, together with the Isomorphism Theorem 2.5, ensures that for every algebra $\boldsymbol{A}$, the mappings

defined respectively as

$$
\rho^{+}: \mathcal{F} i_{\mathrm{GK} \Pi \infty} \boldsymbol{A} \longleftrightarrow C o_{\mathbb{K} \mathbb{P} \infty} \boldsymbol{A}: \boldsymbol{\tau}^{+}
$$

$$
\boldsymbol{\rho}^{+}(F)=\boldsymbol{\Omega}^{\boldsymbol{A}} F \quad \text { and } \quad \boldsymbol{\tau}^{+}(\theta)=1 / \theta
$$

for every $F \in \mathcal{F} i_{\mathbf{G K} \Pi \infty} \boldsymbol{A}$ and $\theta \in C o_{\mathbb{K} \mathbb{P} \infty} \boldsymbol{A}$, are complete lattice isomorphisms with one being the inverse of the other (see for instance [BP89]). We will make use of this isomorphism in the study of the local modal logic, which is not as direct as in the case of $\mathbf{G K} \Pi^{\infty}$.

Concerning the local modal logic $\mathbf{K} \Pi^{\infty}$, we begin by proving a property weaker than being algebraizable.

Lemma 5.5. $\mathbf{K} \Pi^{\infty}$ is equivalential with congruence formulas $\boldsymbol{\Delta}(x, y) \equiv\left\{\square^{n}(x \leftrightarrow y)\right\}_{n \in \omega}$, where $\square^{n} \varphi=\square \square^{n-1} \varphi$ for $n>0$ and $\square^{0} \varphi=\varphi$.

Proof. It suffices to see that $\boldsymbol{\Delta}(x, y)$ meets the fisrt condition of Lemma 2.2 for $\mathbf{K} \Pi^{\infty}$. It is routine to prove this using the already proven completeness of $\mathbf{K} \Pi^{\infty}$ with respect to $\models_{\mathrm{PK}}^{l}$.

This characterization is important for checking that the class of modal product algebras $\mathbb{K P}^{\infty}$ is indeed the algebraic counterpart of $\mathbf{K} \Pi^{\infty}$.

Proposition 5.6. $\operatorname{Alg}^{*} \mathrm{~K} \Pi^{\infty}=\mathbb{K}^{\infty}$.

Proof. Let $\boldsymbol{A} \in \mathbb{K P}^{\infty}$. Taking into account that $\left\{1^{\boldsymbol{A}}\right\}$ is a filter of $\mathbf{K} \Pi^{\infty}$ on $\boldsymbol{A}$, by (2) of Lemma 2.2, $\langle a, b\rangle \in \boldsymbol{\Omega}^{\boldsymbol{A}}\left\{1^{\boldsymbol{A}}\right\}$ iff $\boldsymbol{\Delta}(a, b) \subseteq\left\{1^{\boldsymbol{A}}\right\}$. In particular, this implies that $a \leftrightarrow b=1^{\boldsymbol{A}}$, and so, $a=b$. Therefore, $\boldsymbol{\Omega}^{\boldsymbol{A}}\left\{1^{\boldsymbol{A}}\right\}=\operatorname{Id}_{\boldsymbol{A}}$, and thus, $\left\langle\boldsymbol{A},\left\{1^{\boldsymbol{A}}\right\}\right\rangle \in \operatorname{Mod}^{*} \mathbf{K} \Pi^{\infty}$.

For the converse inclusion, we know that $\boldsymbol{A} \in A l g^{*} \mathbf{K} \Pi^{\infty}$ if and only if there exists $F \in \mathcal{F} i_{\mathbf{K} \Pi \infty} \boldsymbol{A}$ for which $\boldsymbol{\Omega}^{\boldsymbol{A}} F=\operatorname{Id}_{\boldsymbol{A}}$. To see that $\boldsymbol{A} \in \mathbb{K} \mathbb{P}^{\infty}$ if $\boldsymbol{A} \in A l g^{*} \mathbf{K} \Pi^{\infty}$ we have to check that the equations and quasiequations that define $\mathbb{K P}^{\infty}$ hold in $\boldsymbol{A}$ as well.

Each equation $\alpha_{i} \approx \beta_{i}$ corresponding to one of the identities that must hold in an algebra belonging to $\mathbb{K} \mathbb{P}^{\infty}$ comes from an associated theorem $\alpha_{i} \leftrightarrow \beta_{i}$ of $\mathbf{K} \Pi^{\infty}$. By the $\left(\mathrm{N}_{\square}\right)$ rule, it follows that $\square^{n}\left(\alpha_{i} \leftrightarrow \beta_{i}\right)$ is also a theorem of the logic for each $n \in \omega$, and so, for any $h \in \operatorname{Hom}(\mathbf{F m}, \boldsymbol{A})$ it holds that $\boldsymbol{\Delta}\left(h\left(\alpha_{i}\right), h\left(\beta_{i}\right)\right) \subseteq F$, with $\boldsymbol{\Delta}$ being the set of congruence formulas of $\mathbf{K} \Pi^{\infty}$ defined in the previous lemma. By (2) of Lemma 2.2, it follows that $\left\langle h\left(\alpha_{i}\right), h\left(\beta_{i}\right)\right\rangle \in \Omega^{\boldsymbol{A}} F$ for all $h \in \operatorname{Hom}(\mathbf{F m}, \boldsymbol{A})$, and given that by assumption $\Omega^{\boldsymbol{A}} F=\operatorname{Id}_{\boldsymbol{A}}$, it must hold that $h\left(\alpha_{i}\right)=h\left(\beta_{i}\right)$ for all $h \in \operatorname{Hom}(\mathbf{F m}, \boldsymbol{A})$ (so $\boldsymbol{A} \models \alpha_{i} \approx \beta_{i}$ ). 
For the generalized quasiequations $\left(\mathcal{Q}_{\Delta}\right)$ and $\left(\mathcal{Q}_{\rightarrow}\right)$, the proof is similar using that the filters are closed under the correspondent infinitary rules. We will detail the proof for $\left(\mathcal{Q}_{\Delta}\right)$, the case of $\left(\mathcal{Q}_{\rightarrow}\right)$ is analogous.

First observe that $R_{\Delta}$ can be written as $\{(\bar{c} \rightarrow \varphi) \leftrightarrow \overline{1}\}_{c \in(0,1)_{\mathbb{Q}}} \vdash_{\mathbf{K} \Pi \infty} \varphi \leftrightarrow \overline{1}$. By Theorem 4.4, it follows that for any $n \in \omega,\left\{\square^{n}((\bar{c} \rightarrow \varphi) \leftrightarrow \overline{1})\right\}_{c \in(0,1)_{\mathbb{Q}}} \vdash_{\mathbf{K} \Pi^{\infty}} \square^{n}(\varphi \leftrightarrow \overline{1})$. By definition, filters of $\mathbf{K} \Pi^{\infty}$ are closed under deductions, so for any $a \in A$ and $n \in \omega$,

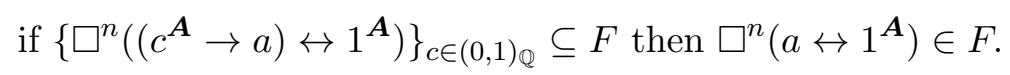

To see that $\mathcal{Q}_{\Delta}$ holds in $\boldsymbol{A}$, let $a \in A$ be such that $c^{\boldsymbol{A}} \rightarrow a=1^{\boldsymbol{A}}$ for all $c \in(0,1)_{\mathbb{Q}}$. Since $\boldsymbol{\Omega}^{\boldsymbol{A}} F=\operatorname{Id}_{\boldsymbol{A}}$, $\left\langle c^{\boldsymbol{A}} \rightarrow a, 1^{\boldsymbol{A}}\right\rangle \in \boldsymbol{\Omega}^{\boldsymbol{A}} F$ for all $c \in(0,1)_{\mathbb{Q}}$. Given that $\mathbf{K} \Pi^{\infty}$ is equivalential, this happens if and only if $\left\{\square^{n}\left(\left(c^{\boldsymbol{A}} \rightarrow a\right) \leftrightarrow 1^{\boldsymbol{A}}\right)\right\}_{c \in(0,1)_{\mathbb{Q}}, n \in \omega} \subseteq F$. From (2) it follows that $\left\{\square^{n}\left(a \leftrightarrow 1^{\boldsymbol{A}}\right)\right\}_{n \in \omega} \subseteq F$, i.e., $\boldsymbol{\Delta}^{\boldsymbol{A}}\left(a, 1^{\boldsymbol{A}}\right) \subseteq F$. Following the same reasoning as before this is equivalent to $\left\langle a, 1^{\boldsymbol{A}}\right\rangle \in \boldsymbol{\Omega}^{\boldsymbol{A}} F$, and so, to $a=1^{\boldsymbol{A}}$ in $\boldsymbol{A}$, which concludes the proof.

We are now able to conclude that, as in the case for the local classical modal logic, $\mathbf{K} \Pi^{\infty}$ is not algebraizable. However, to prove this, we need a different approach than the one used in classical modal logic, where it is enough to define a 4-element Boolean algebra with modalities that is simple but for which there exist more than two filters of the local modal logic. In our case, there do not exist finite $\mathbf{K} \Pi^{\infty}$-algebras, so this ad-hoc construction is more complicated and it is in fact easier to prove it in a more general way. Moreover, since classical logic is not an extension of $\Pi^{\infty}$ (because of the rational constants and the $\Delta$ operator), we cannot use the fact that classical local modal logic is not algebraizable to straightforwardly derive that $\mathbf{K} \Pi^{\infty}$ is not algebraizable either.

Lemma 5.7. $\mathbf{K} \Pi^{\infty}$ is not algebraizable.

Proof. For the sake of a simpler notation, in this proof we let $\mathcal{C} n X=\left\{\theta \in F m: X \vdash_{\mathbf{K} \Pi \infty} \theta\right\}$, and we will write the Leibniz operator $\Omega^{\mathbf{F m}}$ over the Lindenbaum-Tarski algebra $\mathbf{F m}$ of formulas of $\mathbf{K} \Pi^{\infty} F m$ (i.e., the set of formulas modulo $\mathbf{K} \Pi^{\infty}$-equivalence) simply as $\boldsymbol{\Omega}$.

It is clear that for $x, y \in F m$ such that $x \neq y$, we have $\{x, y\} \nvdash_{\mathbf{K} \Pi \infty} \square(x \leftrightarrow y)$, and so, in particular, $\boldsymbol{\Delta}(x, y) \nsubseteq \mathcal{C} n\{x, y\}$. From the definition of equivalential logic and Lemma 5.5 it follows that $\langle x, y\rangle \notin \Omega \mathcal{C} n\{x, y\}$, which in particular proves that $\mathcal{C} n\{x, y\} / \Omega \mathcal{C} n\{x, y\}$ has at least two different elements: $x / \Omega \mathcal{C} n\{x, y\}$ and $y / \Omega \mathcal{C} n\{x, y\}$.

For simplicity, let $\boldsymbol{A}$ denote $\mathbf{F m} / \boldsymbol{\Omega C} n\{x, y\}$. Since $\mathcal{C} n\{x, y\} \in \mathcal{F} i_{\mathbf{K} \Pi \infty \mathbf{F m}}$ it follows that $\boldsymbol{\Omega}^{\boldsymbol{A}}(\mathcal{C} n\{x, y\} / \boldsymbol{\Omega C} n\{x, y\})=\operatorname{Id}_{\boldsymbol{A}}$, and so

$$
\langle\boldsymbol{A}, \mathcal{C} n\{x, y\} / \boldsymbol{\Omega C} n\{x, y\}\rangle \in \operatorname{Mod}^{*} \mathbf{K} \Pi^{\infty},
$$

which implies that $\boldsymbol{A} \in A l g^{*} \mathbf{K} \Pi^{\infty}=\mathbb{K} \mathbb{P}^{\infty}$. We know that $\{\overline{1}\}$ is a filter of $\mathbf{K} \Pi^{\infty}$ over $\boldsymbol{A}$, so

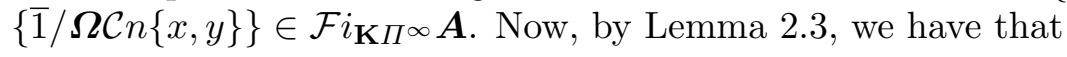

$$
\boldsymbol{\Omega}^{\boldsymbol{A}}\{\overline{1} / \boldsymbol{\Omega C} n\{x, y\}\} \subseteq \boldsymbol{\Omega}^{\boldsymbol{A}}(\mathcal{C} n\{x, y\} / \boldsymbol{\Omega C} n\{x, y\})=\operatorname{Id}_{\boldsymbol{A}},
$$

and so, $\boldsymbol{\Omega}^{\boldsymbol{A}}\{\overline{1} / \boldsymbol{\Omega C} n\{x, y\}\}=\operatorname{Id}_{\boldsymbol{A}}$ as well. Then, both

$$
\langle\boldsymbol{A}, \mathcal{C} n\{x, y\} / \boldsymbol{\Omega C} n\{x, y\}\rangle \quad \text { and } \quad\langle\boldsymbol{A},\{\overline{1} / \boldsymbol{\Omega C} n\{x, y\}\}\rangle
$$

are reduced models of $\mathbf{K} \Pi^{\infty}$. Since their algebraic counterparts coincide, but they are different models (given that $\mathcal{C} n\{x, y\} / \Omega \mathcal{C} n\{x, y\}$ has at least two elements, and $\{\overline{1} / \boldsymbol{\Omega C} n\{x, y\}\}$ is a singleton), we have that $\boldsymbol{\Omega}$ is not injective, and thus, it is not an isomorphism as the Isomorphism Theorem requires.

Recall that $\mathbf{K} \Pi^{\infty}$ is strongly complete with respect to its reduced models. Inspired by [Mal86], where the analogous problem for the classical modal logic is studied, we can give a more specific characterization of this class of models, and thus, obtain a more concrete completeness result for $\mathbf{K} \Pi^{\infty}$ as an immediate corollary. 
Theorem 5.8. $\langle\boldsymbol{A}, F\rangle$ is a reduced model of $\mathbf{K} \Pi^{\infty}$ if and only if $\boldsymbol{A} \in \mathbb{K} \mathbb{P}^{\infty}, F \in \mathcal{F} i_{\mathbf{K} \Pi \infty} \boldsymbol{A}$ and $\left\{1^{A}\right\}$ is the only open filter included in $F$.

Proof. For the left to right direction, let $\langle\boldsymbol{A}, F\rangle \in \operatorname{Mod}^{*} \mathbf{K} \Pi^{\infty}$. From Lemma 5.6 it follows that $\boldsymbol{A} \in \mathbb{K P}^{\infty}$ and it also holds that $F \in \mathcal{F} i_{\Pi^{\infty}}(\overline{\boldsymbol{A}})$. By way of contradiction, suppose there is an open $\mathbf{K} \Pi^{\infty}$-filter $G$ on $\boldsymbol{A}$ such that $\{1\} \varsubsetneqq G \subseteq F$. Since both $\{1\}$ and $G$ are open filters, they are also deductive $\mathbf{G K} \Pi^{\infty}$-filters on $\boldsymbol{A}$. Given that $\mathbf{G K} \Pi^{\infty}$ is algebraizable, $\boldsymbol{\Omega}^{\boldsymbol{A}}$ is a lattice isomorphism between $\mathcal{F} i_{\mathbf{G K} \Pi^{\infty}} \boldsymbol{A}$ and $C_{\mathbb{K K}_{\mathbb{P}} \infty} \boldsymbol{A}$, and, in particular, $\boldsymbol{\Omega}^{\boldsymbol{A}}: \mathcal{F} i_{\mathrm{GK} \Pi \infty} \boldsymbol{A} \rightarrow C_{\mathbb{K}_{\mathbb{P}} \infty} \boldsymbol{A}$ is injective. Then, given that $\{1\} \neq G, \boldsymbol{\Omega}^{\boldsymbol{A}}\{1\} \neq \boldsymbol{\Omega}^{\boldsymbol{A}} G$. It is clear that $\boldsymbol{\Omega}^{\boldsymbol{A}}\{1\}=\operatorname{Id}_{\boldsymbol{A}}$ and so, $\boldsymbol{\Omega}^{\boldsymbol{A}} G \neq \operatorname{Id}_{\boldsymbol{A}}$. $\mathbf{K} \Pi^{\infty}$ is equivalential so $\boldsymbol{\Omega}^{\boldsymbol{A}}$ is monotone over the $\mathbf{K} \Pi^{\infty}$-filters on $\boldsymbol{A}$. Given that both $F$ and $G$ are $\mathbf{K} \Pi^{\infty}$-filters on $\boldsymbol{A}$ it follows that $\operatorname{Id}_{\boldsymbol{A}} \neq \boldsymbol{\Omega}^{\boldsymbol{A}} G \subseteq \boldsymbol{\Omega}^{\boldsymbol{A}} F$, so $\boldsymbol{\Omega}^{\boldsymbol{A}} F \neq \operatorname{Id}_{\boldsymbol{A}}$ which contradicts the assumption that $\langle\boldsymbol{A}, F\rangle \in \operatorname{Mod}^{*} \mathbf{K} \Pi^{\infty}$.

As for the other direction, let $F \in \mathcal{F} i_{\mathbf{K} \Pi \infty} \boldsymbol{A}$ and suppose that $\boldsymbol{\Omega}^{\boldsymbol{A}} F \neq \operatorname{Id}_{\boldsymbol{A}}$. We know that $\boldsymbol{\Omega}^{\boldsymbol{A}}: \mathcal{F} i_{\mathrm{GK} \Pi^{\infty}} \boldsymbol{A} \rightarrow C o_{\mathbb{K} \mathbb{P} \infty} \boldsymbol{A}$ is an isomorphism, and also that $\boldsymbol{\Omega}^{\boldsymbol{A}} F \in C o_{\mathbb{K} \mathbb{P} \infty} \boldsymbol{A}$. Then, there is $G \in \mathcal{F} i_{\mathrm{GK} \Pi \infty} \boldsymbol{A}$ such that $\boldsymbol{\Omega}^{\boldsymbol{A}} G=\boldsymbol{\Omega}^{\boldsymbol{A}} F$, and so, $\boldsymbol{\Omega}^{\boldsymbol{A}} G \neq \operatorname{Id}_{\boldsymbol{A}}$. For any $a \in G,\langle a, 1\rangle \in \boldsymbol{\Omega}^{\boldsymbol{A}} G$ and so $\langle a, 1\rangle \in \boldsymbol{\Omega}^{\boldsymbol{A}} F$ too. Since $1 \in F$ and $\boldsymbol{\Omega}^{\boldsymbol{A}} F$ is congruent with $F$ (by definition), $a \in F$, and so $G \subseteq F$, which concludes the proof.

Finally, the existence and behaviour of the constants in the algebras in $\mathbb{K}^{\infty}$ leads to a very clear characterization of all the linearly ordered modal product algebras.

Theorem 5.9. If an algebra $\boldsymbol{A}=\left\langle A, \odot, \rightarrow, \Delta, \square, \diamond,\left\{c^{\boldsymbol{A}}\right\}_{c \in[0,1]_{\mathbb{Q}}}\right\rangle \in \mathbb{K}^{\infty}$ is linearly ordered then one of the following conditions hold:

- $\square=\diamond=I d_{\boldsymbol{A}}$ (the identity function in $\boldsymbol{A}$ );

- $\square=1^{\boldsymbol{A}}$ and $\diamond=0^{\boldsymbol{A}}$ (the constant functions of value 1 and 0 respectively).

Proof. First we can easily see that for any $a \in A$ both $a \leqslant \square a$ and $\diamond a \leqslant a$ hold. Indeed, if $\square a<a$, from Corollary 3.12, it would follow that there is $c \in[0,1]_{\mathbb{Q}}$ such that $\square a<c^{\boldsymbol{A}}<a$. In that case, it is clear that $\square\left(c^{\boldsymbol{A}} \rightarrow a\right)=1$, but at the same time, $1>c^{\boldsymbol{A}} \rightarrow \square a=\square\left(c^{\boldsymbol{A}} \rightarrow a\right)$, which is a contradiction. For $\diamond$, the proof is analogous.

Now, we will prove by cases that if the interpretation of $\square$ or $\diamond$ is not the identity function, then $\square=\overline{1}$ and $\diamond=\overline{0}$. First, suppose that $\square$ is not the identity function. Since for all $a \in A$ it holds that $a \leqslant \square a$, then there must exist $b \in A$ such that $b<\square b$ (A is a chain by assumption). By Corollary 3.12 there exists $c \in[0,1]_{\mathbb{Q}}$ such that $b<c^{\boldsymbol{A}}<\square b$, from where it follows that $1=c^{\boldsymbol{A}} \rightarrow \square b=\square\left(c^{\boldsymbol{A}} \rightarrow b\right)=\Delta \square\left(c^{\boldsymbol{A}} \rightarrow b\right)$. Given that $\Delta$ and $\square$ commute in $\boldsymbol{A}$, it follows that $\square \Delta\left(c^{\boldsymbol{A}} \rightarrow b\right)=1$. Now note that $\left(c^{\boldsymbol{A}} \rightarrow b\right)<1$ by assumption, and being $\boldsymbol{A}$ a product chain, $\Delta\left(c^{\boldsymbol{A}} \rightarrow b\right)=0$. Then $1=\square \Delta\left(c^{\boldsymbol{A}} \rightarrow b\right)=\square 0$, and using that $\square$ is an increasing function ${ }^{9}$ we can conclude that $\square=\overline{1}$ (since 0 is the minimum element of $\boldsymbol{A}$ ). Given that $\square \neg x=\neg \diamond x$, it is immediate that $\diamond=\overline{0}$.

On the other hand, suppose that for some $b \in A, \diamond b<b$, and so $\diamond b<c^{\boldsymbol{A}}<b$ for some $c \in[0,1]_{\mathbb{Q}}$. It follows that $1=\diamond b \rightarrow c^{\boldsymbol{A}}=\square\left(b \rightarrow c^{\boldsymbol{A}}\right)$, and an analogous reasoning as before can be followed to see that $\square 0=1$. From there, again as above, one can prove that $\square=\overline{1}$ and $\diamond=\overline{0}$, and this concludes the proof.

With this it is clear that neither $\mathbf{K} \Pi^{\infty}$ nor $\mathbf{G K} \Pi^{\infty}$ are complete with respect to $\mathbb{K} \mathbb{P}^{\infty}$-chains. Indeed, from the previous lemma, $\square \overline{0} \vee(\varphi \leftrightarrow \square \varphi)$ is valid in all linearly ordered modal product algebras, but clearly it is not a theorem of $\mathbf{K} \Pi^{\infty}$.

5.2. Complex algebras and Canonical models. Since we have developed two semantics for our modal logics, namely Kripke and algebraic semantics, it is natural to study their relationship. Complex algebras are a family of algebras that arise from Kripke frames through a dual construction,

\footnotetext{
${ }^{9}$ It follows from $\left(\mathrm{E}_{\mathrm{K}}\right)$ that for any $x, y \in A$, if $x \leqslant y$ then $\square x \leqslant \square y$
} 
and were originally defined in the context of classical modal logic (see for instance [CZ97]). The construction method can be easily generalized to the case of many-valued logics, taking into account the fact that the algebra over which the Kripke model evaluates formulas takes an explicit role in the construction of the dual algebra from the model.

In our case, we will describe a way of translating the Kripke semantics into the algebraic one by associating to each product Kripke model a $\mathbf{K} \Pi^{\infty}$-algebra and an evaluation over it.

Remember that for two sets $A, B, A^{B}$ denotes the set of maps from $B$ to $A$. For simplicity we will sometimes denote by $[b \mapsto f(b)]$ the element $f \in A^{B}$ that sends $b \in B$ to $f(b) \in A$.

Definition 5.10. Let $\boldsymbol{A} \in \mathbb{P}^{\infty}$ and $\mathfrak{M}=\langle W, R, e\rangle$ be a (safe) $\boldsymbol{A}$-Kripke model. The complex algebra associated to $\mathfrak{M}$ is the partial algebra

$$
\mathbb{A} l g(\mathfrak{M})=\left\langle A^{W}, \odot, \rightarrow, \Delta, \square, \diamond,\left\{c^{\mathbb{A l g}(\mathfrak{M})}\right\}_{c \in[0,1]_{\mathbb{Q}}}\right\rangle,
$$

where for every $f, g \in A^{W}$ the non-modal operations $\odot, \rightarrow, \Delta$ and $\left\{c^{\mathbb{A} l g(\mathfrak{M})}\right\}_{c \in[0,1]_{\mathbb{Q}}}$ are defined component-wise from the ones of $\mathbf{A}$ and the modal operations are given by

$$
\square f:=[v \mapsto \inf \{f(w): w \in W, R v w\}] \quad \diamond f:=[v \mapsto \sup \{f(w): w \in W, R v w\}] .
$$

The associated evaluation over $\mathbb{A} l g(\mathfrak{M})$ is $e_{\mathfrak{M}}: F m \rightarrow A^{W}$ with

$$
e_{\mathfrak{M}}(\varphi)=[v \mapsto e(v, \varphi)]
$$

Notice that, although the algebra may be partial in the sense that $\square f$ and $\diamond f$ may be not defined for all $f \in A^{W}$, the evaluation $e_{\mathfrak{M}}(\varphi)$ is defined over every formula $\varphi$ because we assume the model $\mathfrak{M}$ to be safe.

It is routine to see that for any product Kripke model $\mathfrak{M}$, its complex algebra $\mathbb{A} l g(\mathfrak{M})$ is a $\mathbf{K} \Pi^{\infty}$ algebra. Also, it is easy to check that the evaluation associated to $\mathfrak{M}$ is indeed a modal evaluation over $\mathbb{A} l g(\mathfrak{M})\left(\right.$ i.e., $\left.e_{\mathfrak{M}} \in \operatorname{Hom}(\mathbf{F m}, \mathbb{A} l g(\mathfrak{M}))\right)$ and that it coincides with the homomorphism built from the mapping that sends each propositional variable $x$ to $[v \mapsto e(v, x)]$.

The previous construction provides us with several concrete modal product algebras, since the class of Complex Algebras is included in $\mathbb{K} \mathbb{P}^{\infty}$. Moreover, using the completeness result of $\mathbf{K} \Pi^{\infty}$ with respect to $\models_{\text {PK }}^{l}$, and in particular, with respect to the canonical Kripke model $\mathfrak{M}_{\mathfrak{c}}$, we will obtain another interesting algebraic completeness result for this logic.

The intuition behind the algebraic completeness result that can be achieved following this approach is as follows. For $\Gamma \cup\{\varphi\} \subseteq F m$ it holds that $\Gamma \vdash_{\mathbf{K} \Pi \infty} \varphi$ if and only if for all $w \in W_{c}$ for which $w([\Gamma]) \subseteq\{1\}$ it holds $w(\varphi)=1$ as well. This condition is easily expressible within the associated complex algebra $\mathbb{A l} g\left(\mathfrak{M}_{\mathfrak{c}}\right)$ : it means that for all $w \in W_{c}$ for which $e_{\mathfrak{M}_{\mathfrak{c}}}([\Gamma])(w) \subseteq\{1\}$ then $e_{\mathfrak{M}_{\mathfrak{c}}}(\varphi)(w)=1$. Given that the $\Delta$ operator is defined on the complex algebras component-wise, it is clear that for any $w \in W_{c}$ and for any formula $\psi$ either $e_{\mathfrak{M}_{\mathfrak{c}}}(\Delta \psi)(w)=1$ or $e_{\mathfrak{M}_{\mathfrak{c}}}(\Delta \psi)(w)=0$. Then, the previous condition can be rewritten as inf $e_{\mathfrak{M}_{\mathfrak{c}}}([\Delta \Gamma]) \leqslant e_{\mathfrak{M}_{\mathfrak{c}}}(\varphi) .{ }^{10}$

Given a product algebra $\boldsymbol{A}$, we will say that $b \in \boldsymbol{A}$ is a Boolean element whenever there exists an element $b^{\prime} \in \boldsymbol{A}$ called complement of $b$ such that $b \vee b^{\prime}=1$ and $b \wedge b^{\prime}=0$. We will denote the set of boolean elements of $\boldsymbol{A}$ by $\mathfrak{B}_{\boldsymbol{A}}$.

Remark 5.11. Given $\boldsymbol{A} \in \mathbb{P}^{\infty}$, observe that $\Delta a \in \mathfrak{B}_{\boldsymbol{A}}$ for any $a \in \boldsymbol{A}$ and that for any $b \in \mathfrak{B}_{\boldsymbol{A}}$ it holds that $b \odot x=b \wedge x$ for any $x \in \boldsymbol{A}$.

The first statement is direct by definition. As for the second, we have $b \wedge x=b \odot(b \rightarrow x)$ by definition. We know from [HGE96, (12) of Lemma 2]) that for any $z \in \boldsymbol{A}$ it holds that $(b \rightarrow x) \leqslant b \vee z \rightarrow x \vee z$. Letting then $z$ be the complement of $b$, we have that $b \wedge x \leqslant b \odot\left(x \vee b^{\prime}\right)$ (since $b \vee b^{\prime}=1$ ), and thus, by commutativity of $\odot$ and $\vee$ in BL (see for instance [Háj98]), $b \wedge x \leqslant(b \odot x) \vee\left(b \odot b^{\prime}\right)=b \odot x\left(\right.$ since $\left.b \wedge b^{\prime}=0\right)$.

\footnotetext{
$10 \leqslant$ is defined component-wise, and thus for each $w \in W_{c}$, either $e_{\mathfrak{M}_{\mathfrak{c}}}([\Delta \Gamma])(w) \subseteq 1$, in which case by assumption $e_{\mathfrak{M}_{\mathfrak{c}}}(\varphi)(w)=1$, or there is $\gamma \in \Gamma$ with $e_{\mathfrak{M}_{\mathfrak{c}}}(\Delta \gamma)(w)=0$, and thus inf $e_{\mathfrak{M}_{\mathfrak{c}}}([\Delta \Gamma])=0$.
} 
Now, with the aim at generalizing the above idea of logic preserving the Boolean degrees of truth to the whole class of $\mathbf{K} \Pi^{\infty}$-algebras, we will refer to the usual definition of logic preserving degrees of truth (cf. $\left[\mathrm{BEF}^{+} 09\right.$, Def. 2.1]).

Definition 5.12. Let $\Gamma \cup\{\varphi\} \subseteq F m, \mathbb{K} \cup\{\boldsymbol{A}\} \subseteq \mathbb{K P}^{\infty}$ and $h \in \operatorname{Hom}(\mathbf{F m}, \boldsymbol{A})$.

- $\Gamma \models \underset{\boldsymbol{A}, h}{\leqslant} \varphi$ if for any $a \in A$, if $a \leqslant h(\gamma)$ for all $\gamma \in \Gamma$ then $a \leqslant h(\varphi)$.

- $\Gamma \models \underset{\boldsymbol{A}}{\leqslant} \varphi$ if $\Gamma \models \underset{\boldsymbol{A}, h}{\leqslant} \varphi$ for any $h \in \operatorname{Hom}(\mathbf{F m}, \boldsymbol{A})$.

- $\Gamma \models \underset{\mathbb{K}}{\leqslant} \varphi$ if $\Gamma \models \underset{\mathbf{B}}{\leqslant} \varphi$ for each $\mathbf{B} \in \mathbb{K}$.

In particular, if $\mathbf{A}$ is a complete algebra, the first definition is equivalent to say that $\inf \{h(\Gamma)\} \leqslant h(\varphi)$.

Now, a second algebraic completeness result for $\mathbf{K} \Pi^{\infty}$ can be obtained just checking the soundness of $\mathbf{K} \Pi^{\infty}$ with respect to the $=\underset{\mathbb{K} \mathbb{P} \infty}{\leqslant}$ logic defined above in which just the preservation for Boolean elements is required.

Theorem 5.13 (Algebraic completeness of $\mathbf{K} \Pi^{\infty}$ ). For any set of modal formulas $\Gamma \cup\{\varphi\}$,

$$
\Gamma \vdash_{\mathbf{K} \Pi \infty} \varphi \text { iff } \Delta \Gamma \models_{\mathbb{K} \mathbb{P} \infty}^{\leqslant} \varphi
$$

where $\Delta \Gamma$ stands for the set $\{\Delta \gamma: \gamma \in \Gamma\}^{11}$

Proof. Completeness is direct because $\mathbb{A} l g\left(\mathfrak{M}_{\mathfrak{c}}\right) \in \mathbb{K}^{\infty}$. If $\Gamma \nvdash_{\mathbf{K} \Pi \infty} \varphi$, by completeness with respect to $\mathfrak{M}_{\mathfrak{c}}$, there exists a world $v \in W_{c}$ such that $v([\Gamma]) \subseteq\{1\}$ and $v(\varphi)<1$. Then we have that $e_{\mathfrak{M}_{\mathfrak{c}}}([\Gamma])(v)=1$ (and so, that $\left.e_{\mathfrak{M}_{\mathfrak{c}}}([\Delta \Gamma])(v)=1\right)$ but $e_{\mathfrak{M}_{\mathfrak{c}}}(\varphi)(v)<1$. But taking into account how the order is defined in $\mathbb{A} l g\left(\mathfrak{M}_{\mathfrak{c}}\right)$, this means that $e_{\mathfrak{M}_{\mathfrak{c}}}([\Delta \Gamma]) \nless e_{\mathfrak{M}_{\mathfrak{c}}}(\varphi)$.

To prove soundness, let $\boldsymbol{A} \in \mathbb{K} \mathbb{P}^{\infty}$ and $h \in \operatorname{Hom}(\mathbf{F m}, \boldsymbol{A})$. Since all axioms of $\mathbf{K} \Pi^{\infty}$ are evaluated to 1 under any homomorphism, we just need to check the soundness of the deduction rules of $\mathbf{K} \Pi^{\infty}$ in $\models \underset{\mathbb{K} \mathbb{P} \infty}{\leqslant}$ :

$(M P) h(\Delta \psi) \wedge h(\Delta(\psi \rightarrow \varphi))=h(\Delta \psi) \odot h(\Delta(\psi \rightarrow \varphi)) \leqslant h(\Delta \psi) \odot(h(\Delta \psi) \rightarrow h(\Delta \varphi)) \leqslant h(\Delta \varphi) \leqslant$ $h(\varphi)$, by the Remark 5.11 and definitions and axioms of product logic.

$\left(G_{\Delta}\right)$ It is direct by definition.

$\left(\mathrm{R}_{\Delta} / \mathrm{R}_{\rightarrow}\right)$ Let $a \in \boldsymbol{A}$ such that $a \leqslant h(\Delta(\bar{c} \rightarrow \varphi))$ for all $c \in(0,1)_{\mathbb{Q}}$. Then, $a \leqslant h(\bar{c} \rightarrow \varphi)=c^{\boldsymbol{A}} \rightarrow h(\varphi)$ for all $c \in(0,1)_{\mathbb{Q}}$. By residuation, $c^{\boldsymbol{A}} \leqslant a \rightarrow h(\varphi)$ for all $c \in(0,1)_{\mathbb{Q}}$, so by $\left(\mathcal{Q}_{\Delta}\right)$ it follows that $a \rightarrow h(\varphi)=1$, i.e., that $a \leqslant h(\varphi)$. The $R \rightarrow$ case can be proved analogously using $(\mathcal{Q} \rightarrow)$.

$\left(N_{\square}\right)$ This rule is only applicable over theorems, so assume that for any modal product algebra, and for any evaluation $e, e(\psi)=1$. Then, by definition of modal product algebra, in particular for any modal morphism $h$, we have $h(\square \psi)=\square h(\psi)=\square 1=1$, so $\models_{\mathbb{K} \mathbb{P} \infty} \square \psi$, and thus, $=\underset{\mathbb{K} \mathbb{P}^{\infty}}{\leqslant} \square \psi$ as well.

It is natural to close this section by showing how to build a product Kripke model from a $\mathbf{K} \Pi^{\infty}$ algebra $\boldsymbol{A}$ and a modal evaluation over it, in an inverse way to the complex algebra construction, and studying the relation between these two constructions. ${ }^{12}$ A suitable choice for the set of worlds of a Kripke model associated to a $\mathbf{K} \Pi^{\infty}$-algebra $\boldsymbol{A}$ are the set of homomorphisms from $\overline{\boldsymbol{A}}$ into $[0,1]_{\Pi}$.

Definition 5.14. Let $\boldsymbol{A} \in \mathbb{K} \mathbb{P}^{\infty}$, and $e \in \operatorname{Hom}(\mathbf{F m}, \boldsymbol{A})$. The canonical $\langle\boldsymbol{A}, e\rangle$-Kripke model is the model

$$
\mathfrak{M o d}(\boldsymbol{A}, e)=\left\langle W_{\boldsymbol{A}}, R_{\boldsymbol{A}}, e_{\boldsymbol{A}}\right\rangle
$$

where:

\footnotetext{
${ }^{11}$ Do not forget that, in general, modal product algebras are not linearly ordered.

${ }^{12}$ It is remarkable that this construction leads to a more abstract proof of completeness of the logic $\mathbf{K} \Pi^{\infty}$ with respect to its correspondent canonical models. It turns out that the canonical model of the Lindenbaum-Tarski formula algebra of the correspondent logic is the canonical model of the logic.
} 
- $W_{\boldsymbol{A}}=\operatorname{Hom}\left(\overline{\boldsymbol{A}},[0,1]_{\Pi}\right)$;

- $R_{\boldsymbol{A}} v w$ if and only if $w(a)=1$ for all $a \in A$ such that $v(\square a)=1$;

- $e_{\boldsymbol{A}}(v, x)=v(e(x))$.

Our aim is to prove that this construction is inverse to that of the complex algebras from product Kripke models, in the sense that applying these constructions one after the other over a structure of the corresponding type produces a new structure $N$ of the same type in which the original one $O$ can be 'embedded' (in the sense that the consequence relation induced by $N$ is a subset of the one induced by $O$ ). That is to say, for an arbitrary set of formulas $\Gamma \cup\{\varphi\}$,

$$
\Gamma \models_{N} \varphi \text { implies that } \Gamma \models_{O} \varphi \text {. }
$$

In order to do this we need first to see that the evaluation $e_{\boldsymbol{A}}$ of the above canonical Kripke model $\mathfrak{M o d}(\boldsymbol{A}, e)$ verifies the Truth Lemma, i.e., $e_{\boldsymbol{A}}(v, \varphi)=v(e(\varphi))$ for any $\varphi \in F m$ and any $e \in \operatorname{Hom}(\mathbf{F m}, \boldsymbol{A})$.

In fact, we can prove a stronger result that only depends on the frame: since $e \in \operatorname{Hom}(\mathbf{F m}, \boldsymbol{A})$, the previous formulation of the Truth Lemma can be proven as just a corollary of seeing that for any $a \in A$, both $v(\square a)=\inf \left\{w(a): R_{\boldsymbol{A}} v w\right\}$ and $v(\diamond a)=\sup \left\{w(a): R_{\boldsymbol{A}} v w\right\}$ are true.

First, observe that a generalization of Lemma 4.8 easily follows from the fact that each $w \in W_{\boldsymbol{A}}$ is a homomorphism from $\overline{\boldsymbol{A}}$ into $[0,1]_{\Pi}$. Indeed, for any $a, b \in \boldsymbol{A}$ and any $v, w \in W_{\boldsymbol{A}}$, if $v(a) \not z w(b)$ then $w(b)<v(a)$, and thus, there is $c \in[0,1]_{\mathbb{Q}}$ such that $w(b)<c<v(a)$, i.e., $v\left(c^{\boldsymbol{A}} \rightarrow a\right)=1$ and $w\left(c^{\boldsymbol{A}} \rightarrow b\right)<1$. Using the equations $\left(\mathrm{E}_{\mathrm{A}_{\square} 1}\right)$ and $\left(\mathrm{E}_{\mathrm{A} \diamond 1}\right)$ we get

- $v(\square a) \leqslant w(a)$ for all $v, w \in W_{\boldsymbol{A}}$ with $R_{\boldsymbol{A}} v w$;

- $v(\diamond a) \geqslant w(a)$ for all $v, w \in W_{\boldsymbol{A}}$ with $R_{\boldsymbol{A}} v w$.

As for the converse inequalities, a more general version of Lemma 4.9 can be proven.

Lemma 5.15. Let $\boldsymbol{A} \in \mathbb{K}^{\infty}$ and $e \in \operatorname{Hom}(\mathbf{F m}, \boldsymbol{A})$. Let $v \in W_{\boldsymbol{A}}$ and $a \in A$. If $w(a)=1$ for all $w \in W_{\boldsymbol{A}}$ such that $R_{\boldsymbol{A}}$ vw then $v(\square a)=1$.

Proof. First, it is easy to see that for any $w \in W_{\boldsymbol{A}}$ the following hold:

- $w^{-1}(1)$ is a prime $\Pi^{\infty}$-filter on $\overline{\boldsymbol{A}}$ (recall that $\overline{\boldsymbol{A}}$ is the non-modal reduct of $\boldsymbol{A}$ ) and

- $\square^{-1} w^{-1}(1)=\{a \in A: w(\square a)=1\}$ is a $\Pi^{\infty}$-filter on $\overline{\boldsymbol{A}}$. This is easy to check using that $w$ is an homomorphism from $\overline{\boldsymbol{A}}$ into $[0,1]_{\Pi}$ and that the equations and generalised quasi-equations arising from the axiomatization of $\mathbf{K} \Pi^{\infty}$ hold in $\boldsymbol{A}$. In order to check that $1 \in \square^{-1} w^{-1}(1)$ and that $\square^{-1} w^{-1}(1)$ is closed under $\mathrm{MP},\left(\mathbf{G}_{\Delta}\right), \mathrm{R}_{\Delta}$ and $\mathrm{R}_{\rightarrow}$ it is just necessary to use, respectively, $\left(\mathrm{E}_{\mathrm{K}}\right),\left(\mathrm{E}_{\mathrm{K}}\right),\left(\mathrm{E}_{\mathrm{A}_{\square} 2}\right),\left(\mathrm{E}_{\mathrm{A} \diamond 1}\right)$ and $\left(\mathrm{E}_{\mathrm{A}_{\square} 1}\right)$ from the definition of the class of $\mathbb{K P}^{\infty}$ algebras.

On the other hand, we can prove the following claim:

Claim: The set of prime filters that contain $\square^{-1} v^{-1}(1)$ coincides with the set $\left\{w^{-1}(1)\right.$ : $w \in W_{\boldsymbol{A}}$ and $\left.R_{\boldsymbol{A}} v w\right\}$.

The fact that for each $w \in W_{\boldsymbol{A}}$ such that $R_{\boldsymbol{A}} v w$ it holds that $w^{-1}(1)$ is a prime filter that contains $\square^{-1} v^{-1}(1)$ is immediate by definition. We want to check that each prime filter that contains $\square^{-1} v^{-1}(1)$ coincides with $w^{-1}(1)$ for some $w \in W_{\boldsymbol{A}}$ that is related with $v$. Define for each filter $F$ as before (i.e., prime and containing $\left.\square^{-1} v^{-1}(1)\right)$ the homomorphism $h_{F} \in \operatorname{Hom}\left(\overline{\boldsymbol{A}},[0,1]_{\Pi}\right)$ by letting $h_{F}=\rho_{F} \circ \Pi_{F}$, where $\Pi_{F}: \overline{\boldsymbol{A}} \rightarrow \overline{\boldsymbol{A}} / F$ is the projection over the quotient algebra and $\rho_{F}$ is the embedding from $\overline{\boldsymbol{A}} / F$ into $[0,1]_{\Pi}$ built in the proof of Lemma 3.9 (since $F$ is prime, $\overline{\boldsymbol{A}} / F$ is a $\Pi^{\infty}$-chain). It is clear that $F=h_{F}^{-1}(1)$. To check that $R_{\boldsymbol{A}} v h_{F}$, observe that for any $a \in A$ such that $v(\square a)=1$, by definition $a \in \square^{-1} v^{-1}(1)$, and so, $a \in F$. Then, $\Pi_{F}(a)=[1]_{F}$, and $h_{F}(a)=1$. 
By the previous claim and Lemma 3.5, it follows that $\square^{-1} v^{-1}(1)=\bigcap\left\{w^{-1}(1): w \in W_{\boldsymbol{A}}\right.$ and $\left.R_{\boldsymbol{A}} v w\right\}$. The assumption of the lemma was that $w(a)=1$ for all $w$ such that $R_{\boldsymbol{A}} v w$, and thus $a \in \square^{-1} v^{-1}(1)$, which concludes the proof.

From here it is straighforward to prove the following lemma (using the same reasoning as in the proof of Lemma 4.10), and from which follows the previously mentioned more general Truth Lemma that refers to the evaluation $e_{\boldsymbol{A}}$ of the canonical Kripke model $\mathfrak{M o d}(\boldsymbol{A}, e)$ for a $\mathbf{K} \Pi^{\infty}$-algebra $\mathbf{A}$ and a modal A-evaluation $e$.

Lemma 5.16. Let $\boldsymbol{A}$ be a $\mathbf{K} \Pi^{\infty}$-algebra. For any $a \in A$ and $v \in W_{\boldsymbol{A}}$ the following hold.

- $v(\square a)=\inf \left\{w(a): R_{\boldsymbol{A}} v w\right\} ;$

- $v(\diamond a)=\sup \left\{w(a): R_{\boldsymbol{A}} v w\right\}$.

Corollary 5.17 (Truth Lemma II). Let $\boldsymbol{A}$ be a $\mathbf{K} \Pi^{\infty}$-algebra. For any e $\in \operatorname{Hom}(\mathbf{F m}, \boldsymbol{A})$, any formula $\varphi$ and any $v \in W_{\boldsymbol{A}}, e_{\boldsymbol{A}}(v, \varphi)=v(e(\varphi))$.

It is now possible to study how the compositions of the $\mathfrak{M o d}()$ and the $\mathbb{A} l g()$ functions behave. For the sake of a lighter notation, in what follows, given a product Kripke model $\mathfrak{M}$, we will write $\mathfrak{M}^{\prime}$ to denote the model $\mathfrak{M o d}\left(\mathbb{A} l g(\mathfrak{M}), e_{\mathfrak{M}}\right)$ (its associated canonical model). Analogously, for a given $\boldsymbol{A} \in \mathbb{K} \mathbb{P}^{\infty}$ and any modal $\boldsymbol{A}$-evaluation $e$, we will write $\boldsymbol{A}^{\prime}$ instead of $\mathbb{A} l g(\mathfrak{M o d}(\boldsymbol{A}, e)$ ) (the complex algebra associated with $\boldsymbol{A}$ ). Note that the modal evaluation $e$ does not affect the resulting algebra, since for any two evaluations $e_{1}, e_{2}, \mathbb{A} l g\left(\mathfrak{M o d}\left(\boldsymbol{A}, e_{1}\right)\right)=\mathbb{A} l g\left(\mathfrak{M o d}\left(\boldsymbol{A}, e_{2}\right)\right)$.

Lemma 5.18. Let $\boldsymbol{A} \in \mathbb{P}^{\infty}$, let $\mathfrak{M}=\langle W, R$, e $\rangle$ be an $\boldsymbol{A}$-Kripke model and let $\Gamma \cup\{\varphi\} \subseteq F m$. Then

$$
\Gamma \forall_{\mathfrak{M}}^{l} \varphi \text { implies } \Gamma \nvdash_{\mathfrak{M}^{\prime}}^{l} \varphi \text {. }
$$

Proof. Suppose there is $w \in W$ such that $e(w, \Gamma) \subseteq\{1\}$ and $e(w, \varphi)<1$. Then, $e_{\mathfrak{M}}(\Gamma) \subseteq F$ and $e_{\mathfrak{M}}(\varphi) \notin F$, where $F$ is the $\Pi^{\infty}$-filter on $\overline{\mathbb{A} l g(\mathfrak{M})}$ defined by $F:=\left\{f \in A^{W}: f(w)=1\right\}$. By Lemma 3.5 , there exists a prime filter $F_{p}$ that contains $F$ (and thus, $\left.e_{\mathfrak{M}}(\Gamma)\right)$ and does not contain $e_{\mathfrak{M}}(\varphi)$. Consider then the world $h \in W^{\prime}=\operatorname{Hom}\left(\overline{\mathbb{A} l g(\mathfrak{M})},[0,1]_{\Pi}\right)$ given by $h=\rho_{F_{p}} \circ \Pi_{F_{p}}$, where $\rho_{F_{p}}$ is the complete embedding defined in Theorem 3.9 and $\Pi_{F_{p}}$ is the projection from $\boldsymbol{A}$ to $\boldsymbol{A} / F_{p}$. Then, using Lemma 5.16 we get that $e^{\prime}(h, \Gamma)=h\left(e_{\mathfrak{M}}(\Gamma)\right) \subseteq\{1\}$ and $e^{\prime}(h, \varphi)=h\left(e_{\mathfrak{M}}(\varphi)\right)<1$.

The converse of this lemma is not true in general. It is very easy to build a counterexample for $\boldsymbol{A}$ being the direct product of the canonical product algebra by itself, i.e., $\boldsymbol{A}=[0,1]_{\Pi} \times[0,1]_{\Pi}$. Just consider a model $\mathfrak{M}$ with one world $w$ and no relations, and such that $e(w, p)=\langle 0,1\rangle$ and $e(w, q)=\langle 1,0\rangle$. Clearly, $p \models_{\mathfrak{M}}^{l} q$, but $p \forall_{\mathfrak{M}^{\prime}}^{l} q$, considering the evaluation on the world $h \in \operatorname{Hom}\left([0,1]_{\Pi} \times[0,1]_{\Pi},[0,1]_{\Pi}\right)$ such that $h^{-1}(1)=\{\langle x, 1\rangle: x \in[0,1]\}$.

Nevertheless, we can prove that when the algebra $\boldsymbol{A}$ is linearly ordered, the previous lemma can be generalized to an equivalence result. For simplicity, given a product algebra $\boldsymbol{A}$ and an arbitrary set $W$, we will denote by $\boldsymbol{A}^{W}$ the product algebra of universe $A^{W}$ and with its operations defined component-wise.

Lemma 5.19. Let $\boldsymbol{A} \in \mathbb{P}^{\infty}$ be linearly ordered, $W$ an arbitrary denumerable set and $F$ a linear $\Pi^{\infty}$-filter on $\boldsymbol{A}^{W}$. Then there exists $I \subseteq W$ such that $F=\left\{f \in A^{W}: f(w)=1\right.$ for all $\left.w \in I\right\}$.

Proof. Let $I:=\bigcap_{f \in F}\{w \in W: f(w)=1\}$, and $B=\left\{g \in A^{W}: g(w)=1\right.$ for all $\left.w \in I\right\}$. To see that $F \subseteq B$, pick any $f \in F$, and notice that for any $v \in W$ such that $f(v)<1, v \notin I$. Then, it is clear that $f \in B$. On the other hand, to prove that $B \subseteq F$, let $b \in B$. It is straightforward to see that, for any $x \in A^{W}$, either $\Delta x \in F$ or $\neg \Delta x \in F$, so in particular, either $\Delta b \in F$ or $\neg \Delta b \in F$. Towards a contradiction, suppose that $\neg \Delta b \in F$. Then, given that for all $v \in I, b(v)=1$, it holds that $(\neg \Delta b)(v)=0$, so by definition of $I$ in terms of the elements in $F, v \notin I$, which is a contradiction. Then, $\Delta b \in F$, and since the equality $\Delta x \rightarrow x=1$ holds in $\mathbb{P}^{\infty}$, it implies that $b \in F$ as well, concluding the proof. 
Theorem 5.20. Let $\boldsymbol{A} \in \mathbb{P}^{\infty}$ be linearly ordered, $\mathfrak{M}=\langle W, R, e\rangle$ be an $\boldsymbol{A}$-Kripke model and $\Gamma \cup\{\varphi\} \subseteq F m$. Then

$$
\Gamma \models_{\mathfrak{M}}^{l} \varphi \text { iff } \Gamma \models_{\mathfrak{M}^{\prime}}^{l} \varphi \text {. }
$$

Proof. The right to left direction was proven in general in Lemma 5.18. To prove the other direction, suppose there is $h \in \operatorname{Hom}\left(\overline{\mathbb{A} l g(\mathfrak{M})},[0,1]_{\Pi}\right)$ such that $e^{\prime}(h, \Gamma) \subseteq\{1\}$ and $e^{\prime}(h, \varphi)<1$. By Lemma 5.16, and letting $F$ be the linear filter given by the set $h^{-1}(1)$, we know that $[v \mapsto e(v, \gamma)] \in F$ for all $\gamma \in \Gamma$ and $[v \mapsto e(v, \varphi)] \notin F$. By the previous lemma, there exists $I \subseteq W$ such that $F=\left\{f \in A^{W}: f(w)=1\right.$ for all $\left.w \in I\right\}$. Then, $[v \mapsto e(v, \gamma)] \in F$ for all $\gamma \in \Gamma$ is equivalent to say that $e(w, \gamma)=1$ for all $w \in I$ and all $\gamma \in \Gamma$. On the other hand, we have that $[v \mapsto e(v, \varphi)] \notin F$ implies that there is some $w \in I$ such that $e(w, \varphi)<1$, so we have that $\Gamma \forall_{\mathfrak{M}}^{l} \varphi$.

In the case of the global modal logic, it is possible to obtain a more general version of the above result.

Theorem 5.21. Let $\boldsymbol{A} \in \mathbb{P}^{\infty}, \mathfrak{M}=\langle W, R, e\rangle$ be an $\boldsymbol{A}$-Kripke model and $\Gamma \cup\{\varphi\} \subseteq F m$. Then

$$
\Gamma \models_{\mathfrak{M}}^{g} \varphi \text { iff } \Gamma \models_{\mathfrak{M}^{\prime}}^{g} \varphi \text {. }
$$

Proof. First, observe that for any set of formulas $X, \mathfrak{M}$ is a model of $X$ if and only if $\mathfrak{M}^{\prime}$ is a model of $X$. The right to left direction was proved in Lemma 5.18 ( $\mathfrak{M}$ is not a model of $X$ if there is $x \in X$ such that $\left.\emptyset \forall_{\mathfrak{M}}^{l} x\right)$. For the other direction, assume there is $x \in X$ and $h \in \operatorname{Hom}\left(\overline{\mathbb{A} l g(\mathfrak{M})},[0,1]_{\Pi}\right)$ such that $e^{\prime}(h, x)<1$. By definition this means that $h([v \mapsto e(v, x)])<1$ and so, there must exist $v \in W$ such that $e(v, x)<1$ (otherwise, $[v \mapsto e(v, x)]$ would be the top element of $\overline{\mathbb{A} l g(\mathfrak{M})}$ ).

Then, by definition, $\Gamma \models_{\mathfrak{M}}^{g} \varphi$ if either $\mathfrak{M}$ is not a model of $\Gamma$ or if $\mathfrak{M}$ is a model of $\Gamma \cup\{\varphi\}$. By the previous observation, these respectively happen if and only if either $\mathfrak{M}^{\prime}$ is not a model of $\Gamma$ or if $\mathfrak{M}^{\prime}$ is a model of $\Gamma \cup\{\varphi\}$, which concludes the proof.

On the other hand, we can also study the relation between a modal algebra $\boldsymbol{A}$ and its associated complex algebra $\boldsymbol{A}^{\prime}$.

Theorem 5.22. Let $\boldsymbol{A}$ be a $\mathbf{K} \Pi^{\infty}$-algebra and e be a modal evaluation in $\boldsymbol{A}$. Then $\boldsymbol{A}$ can be embedded into $\boldsymbol{A}^{\prime}$.

Proof. Note that the universe of the algebra $\mathbb{A} l g(\mathfrak{M o d}(\boldsymbol{A}, e))$ is the set $[0,1]^{\operatorname{Hom}\left(\overline{\boldsymbol{A}},[0,1]_{\Pi}\right)}$. Let $\rho$ be the function that maps each element $a \in A$ to the function from $\operatorname{Hom}\left(\overline{\boldsymbol{A}},[0,1]_{\Pi}\right)$ to $[0,1]$ that sends each $h$ to $h(a)$, i.e., $\rho(a)=[h \mapsto h(a)]$ for each $h \in \operatorname{Hom}\left(\overline{\boldsymbol{A}},[0,1]_{\Pi}\right)$. From the Truth Lemma 5.17 it follows that $\rho$ is a modal homomorphism from $\boldsymbol{A}$ to $\mathbb{A} l g(\mathfrak{M o d}(\boldsymbol{A}, e))$. It is an exercise to see that it is injective.

As a corollary, it is easy to see that for any modal algebra $\boldsymbol{A}$ and any set of formulas $\Gamma \cup\{\varphi\} \subseteq F m$

$$
\Delta \Gamma \not{ }_{\boldsymbol{A}}^{\leqslant} \varphi \text { implies that } \Delta \Gamma \not \boldsymbol{A}^{\prime}, \varphi \text {. }
$$

\section{Some Properties of $\mathbf{K} \Pi^{\infty}$}

In this section we will further study the logic $\mathbf{K} \Pi^{\infty}$ in different aspects, presenting a miscellanea of results. We begin by focusing on some interdefinability issues between the modal operators of the logic, then we consider axiomatic extensions of $\mathbf{K} \Pi^{\infty}$ with some usual modal axioms and their corresponding Kripke models. We finish by studying a possibilistic product logic, its embedding into an S5-type extension of $\mathbf{K} \Pi^{\infty}$, and its expansion with an involutive negation. 
6.1. Interdefinability of $\square$ and $\diamond$. As we remarked in Section 2, a very interesting fact of having rational constants in the language of $\mathbf{K} \Pi^{\infty}$ is that of obtaining a very strong relation between the $\diamond$ and the $\square$ operators. Even though they are not completely interdefinable, as it happens when the logic has an involutive negation, it is possible to use the constants to define a negation that will partially behave in an involutive way. This will allows us to show a partial inter-definability between the modal operators.

For any $c \in[0,1]_{\mathbb{Q}}$, let us consider a new unary connective $\neg_{c}$ defined as $\neg_{c} \varphi:=\varphi \rightarrow \bar{c}$. These connectives are a sort of generalized negation, in fact $\neg_{\mathbf{0}}=\neg$, and e.g. they were already used by Jenei in [Jen05] to study the question of determining the product t-norm (and others) by some level sets of its graph. Clearly, whenever $e(\varphi) \geqslant c>0$ or $e(\varphi) \in\{0,1\}$ and $c=0, \neg_{c}$ behaves in an involutive way over $\varphi$. That is to say, for $c \in(0,1] \neg_{c}$ is an involutive operator in the range [c,1] of the $[0,1]_{\Pi}$ algebra (while, as it was already known, $\neg_{0}=\neg$ is only involutive over $\{0,1\}$, and $\neg_{1}$ is trivially involutive only over 1 ). By completeness of $\Pi^{\infty}$, this amounts to express that for all $c>0$, the following formula is a theorem of $\Pi^{\infty}$ (and so, of $\mathbf{K} \Pi^{\infty}$ too):

$$
\Delta(\bar{c} \rightarrow \varphi) \rightarrow\left(\neg_{c} \neg_{c} \varphi \leftrightarrow \varphi\right) .
$$

It is easy to see (semantically) that, for any constant, and in particular, for $c=0, \square \neg_{c} \varphi \leftrightarrow \neg_{c} \diamond \varphi$ is a theorem of $\mathbf{K} \Pi^{\infty}$. Then, whenever $\neg_{c}$ behaves in an involutive way over $\diamond \varphi$, it is clear that it is possible to (locally) express $\diamond$ in terms of the $\square$ operator, and viceversa. This is shown in next lemma.

Lemma 6.1. Let $\varphi$ be a modal formula, and $v$ an arbitrary world of the canonical model. Then there exist $c, d \in[0,1]_{\mathbb{Q}}$ such that

(1) $v(\diamond \varphi)=v\left(\neg_{c} \square \neg_{c} \varphi\right)$

(2) if $v(\square \varphi)>0$ then $v(\square \varphi)=v\left(\neg_{d} \diamond \neg_{d} \varphi\right)$

Proof. (1) We can split the proof in two. First, if $v(\diamond \varphi)=0$, let $c=0$ too, and observe that $v(\neg \square \neg \varphi)=\neg \neg v(\diamond \varphi)=\neg \neg 0=0$. If $v(\diamond \varphi)>0$, then pick $0<c<v(\diamond \varphi)$. Then, $v\left(\neg_{c} \square \neg_{c} \varphi\right)=(v(\diamond \varphi) \rightarrow c) \rightarrow c=c /(c / v(\diamond \varphi))=v(\diamond \varphi)$.

(2) $v(\square \varphi)>0$ implies that there exists $0<d<v(\square \varphi)$, and by definition, $d<w(\varphi)$ for all $w \in W_{\mathfrak{c}}$ such that $R_{\mathfrak{c}} v w$. Then for all the worlds $w$ as before it holds that $w\left(\neg d \neg{ }_{d} \varphi\right)=w(\varphi)$. Using axiom $\left(\mathrm{A}_{\diamond} 1\right)$ we immediately get $v\left(\neg_{d} \diamond \neg_{d} \varphi\right)=v\left(\square \neg_{d} \neg d \varphi\right)=v(\square \varphi)$.

It is interesting to notice that the second statement in the above lemma is just a reformulation of the quasi-witnessed model property for predicate Product logic, adapted to the particular case of the Product modal logic (see for instance [CE11] for more details at this respect).

A more general statement can be given by adding a precondition ensuring the involutive behaviour of the negations $\neg_{c}$.

Theorem 6.2. The following are theorems of $\mathbf{K} \Pi^{\infty}$ for any constant $c \in(0,1)_{\mathbb{Q}}$.

(1) $\neg \Delta \square \neg_{c} \varphi \rightarrow\left(\diamond \varphi \leftrightarrow \neg_{c} \square \neg_{c} \varphi\right)$;

(2) $\neg \diamond \Delta \neg_{c} \varphi \rightarrow\left(\square \varphi \leftrightarrow \neg_{c} \diamond \neg_{c} \varphi\right)$;

Proof. (1) For $v \in W_{\mathfrak{c}}, v(\neg \Delta \square \neg \varphi)=1$ whenever $v\left(\Delta \square \neg_{c} \varphi\right)=0$, and $v\left(\neg \Delta \square \neg_{c} \varphi\right)=0$ otherwise, in which case the formula is trivially true. $v\left(\Delta \square \neg_{c} \varphi\right)=0$ if and only if $v\left(\square \neg_{c} \varphi\right)<1$ and from axiom $\left(A_{\diamond} 1\right)$ it follows that $v\left(\neg_{c} \diamond \varphi\right)<1$. Using that in this case, $\neg_{c}$ is involutive we conclude that $v\left(\neg_{c} \square \neg_{c} \varphi\right)=v\left(\neg_{c} \neg_{c} \diamond \varphi\right)=v(\diamond \varphi)$.

(2) Similarly, for $v \in W_{\mathfrak{c}}, v\left(\neg \diamond \Delta \neg_{c} \varphi\right)=1$ whenever $v\left(\diamond \Delta \neg_{c} \varphi\right)=0$, and it equals 0 in any other case. $v\left(\diamond \Delta \neg_{c} \varphi\right)=0$ if and only if $w\left(\Delta \neg_{c} \varphi\right)=0$ for all $w \in W_{\mathfrak{c}}$ such that $R_{\mathfrak{c}} v w$, and so, if and only if $w\left(\neg_{c} \varphi\right)<1$ for any $w \in W_{\mathfrak{c}}$ such that $R_{\mathfrak{c}} v w$. Then, it holds that $w\left(\neg_{c} \neg_{c} \varphi\right)=w(\varphi)$ for any $w \in W_{\mathfrak{c}}$ such that $R_{\mathfrak{c}} v w$. Thus $v\left(\neg_{c} \diamond \neg_{c} \varphi\right)=v\left(\square\left(\neg_{c} \neg_{c} \varphi\right)\right)=v(\square \varphi)$. 
6.2. Some usual extensions of the minimal modal logic $\mathbf{K} \Pi^{\infty}$. We can check there is, as expected, a correspondence between extensions of $\mathbf{K} \Pi^{\infty}$ with common modal axioms and their corresponding classes of product Kripke models.

First, we will recall the main properties that are generally studied over accessibility relations, which allow the use of the adequate class of structures to treat a certain problem.

Definition 6.3. Let $R$ be a binary crisp relation on $W$. We say that $R$ is

- Serial iff for any $v \in W$ there is $w \in W$ such that $R v w(\mathcal{D})$;

- Reflexive iff $R v v$ for every $v \in W(\mathcal{T})$;

- Symmetric iff $R v w$ implies that $R w v$ for all $v, w \in W(\mathcal{B})$;

- Euclidean iff for any $v, w, z \in X$ such that $R v w$ and $R v z$ it holds that $R w z(5)$;

- Transitive iff for any $v, w, z \in W$ such that $R v w$ and $R w z$ it holds that $R v z$ (4).

We will say that a model enjoys a set of properties whenever its accessibility relation does so. For each $p \in\{\mathcal{D}, \mathcal{T}, \mathcal{B}, 5,4\}$, we will write $\mathrm{PK}^{p}$ to denote the class of product Kripke models that enjoy the property $p$. Then, for $\mathcal{A} \subseteq\{\mathcal{D}, \mathcal{T}, \mathcal{B}, 5,4\}, \mathrm{PK}^{\mathcal{A}}=\bigcap_{p \in \mathcal{A}} \mathrm{PK}^{p}$.

Definition 6.4. We let the main structural axioms be the following set of modal formulas:

(D) $\square \varphi \rightarrow \diamond \varphi$;

(T) $\square \varphi \rightarrow \varphi$;

(B) $\diamond \square \varphi \rightarrow \varphi$;

(5) $\diamond \square \varphi \rightarrow \square \varphi$;

(4) $\square \varphi \rightarrow \square \square \varphi$.

For $A \subseteq\{(\mathrm{D}),(\mathrm{T}),(\mathrm{B}),(5),(4)\}$ we will denote by $\mathbf{K} \Pi^{\infty A}$ the logic obtained by extending $\mathbf{K} \Pi^{\infty}$ with the set of axioms in $A$. It is obvious there is a 1-1 relation between subsets of structural axioms $A$ and subsets of properties $\mathcal{A}$, relating each axiom with its correspondent property over the Kripke models. It is not hard to prove that indeed this relation preserves the completeness of the extensions with respect to the classes of models that enjoy the corresponding properties.

Theorem 6.5. Let $A \subseteq\{(\mathrm{D}),(\mathrm{T}),(\mathrm{B}),(5),(4)\}$ and $\Gamma \cup\{\varphi\} \subseteq F m$. Then $\Gamma \vdash_{\mathrm{K} \Pi \infty A} \varphi$ if and only if $\Gamma \models{ }_{\mathrm{PK} \mathcal{A}}^{l} \varphi$.

Proof. It is enough to define the canonical model $\mathfrak{M}_{c}^{A}$ of $\mathbf{K} \Pi^{\infty A}$ by letting its universe be $W_{\mathfrak{c}}^{\mathcal{A}}:=$

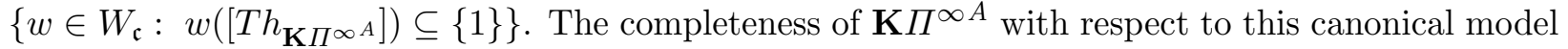
is direct, by an analogous reasoning as that of the one done for $\mathbf{K} \Pi^{\infty}$. To see that the extension is complete with respect to the class of models that enjoy a certain set of properties, it is enough to check that for each axiom in $A$, the canonical model $\mathfrak{M}_{c}^{A}$ enjoys the corresponding property. We will detail two cases, the rest can be done similarly.

(1) Assume $\mathrm{D} \in A$. Suppose towards a contradiction that there is $v \in W_{\mathfrak{c}}^{A}$, for which there is no $w \in W_{\mathfrak{c}}^{A}$ such that $R_{\mathfrak{c}}^{A} v w$. Then, by definition it holds that $v(\square 0)=1$ and $v(\diamond 0)=0$, but this contradicts axiom (D), so $\mathfrak{M}_{c}^{A}$ is serial.

(2) Assume $\mathrm{T} \in A$. Suppose towards a contradiction that there is $v \in W_{\mathfrak{c}}^{A}$ such that it does not hold that $R_{\mathfrak{c}}^{A} v v$, i.e., by definition of the canonical relation, there is $\theta \in F m$ such that $v(\square \theta)=1$ but $v(\theta)<1$. This contradicts axiom $(\mathrm{T})$, so $\mathfrak{M}_{c}^{A}$ is reflexive.

6.3. Possibilistic product logic. It is not in the scope of this paper to study product modal logics defined over non crisp product Kripke Frames, that is, Kripke frames $\langle W, R\rangle$ where the accessibility relation $R$ generalizes to a fuzzy relation valued in an arbitrary product algebra $\boldsymbol{A}$, i.e., relations of the form $R: W \times W \rightarrow \boldsymbol{A}$. The gap between the crisp product modal logics that have been studied in this paper and these non-crisp modal logics is quite big, since there is a lack of general 
tools or approaches in the literature for this subject, with the only exceptions of modal logics over Gödel fuzzy logic ([CR10, CR15]) and over logics with truth-values in a finite residuated lattice [BEGR11]. However, there is a particular kind of non-crisp modal logic over product algebras that can be studied with the tools developed in this paper, namely logics with possibilistic semantics.

Possibilistic logic (see e.g. [DLP94, DP04]) is a well-known uncertainty logic for reasoning with graded beliefs on classical propositions by means of necessity and possiblity measures. Possibilistic logic deals with weighted formulas $(\varphi, r)$, where $\varphi$ is a classical proposition and $r \in[0,1]$ is a weight, interpreted as a lower bound for the necessity degree of $\varphi$. The semantics of these degrees is defined in terms of possibility distributions $\pi: \Omega \rightarrow[0,1]$ on the set $\Omega$ of classical interpretations of a given propositional language. A possibility distribution $\pi$ on $\Omega$ ranks interpretations according to its plausibility level: $\pi(w)=0$ means that $w$ is rejected, $\pi(w)=1$ means that $w$ is fully plausible, while $\pi(w)<\pi\left(w^{\prime}\right)$ means that $w^{\prime}$ is more plausible than $w$. A possibility distribution $\pi: \Omega \rightarrow[0,1]$ induces a pair of dual possibility and necessity measures on propositions, defined respectively as:

$$
\begin{gathered}
\Pi(\varphi):=\sup \{\pi(w) \mid w \in \Omega, w(\varphi)=1\} \\
\mathrm{N}(\varphi):=\inf \{1-\pi(w) \mid w \in \Omega, w(\varphi)=0\} .
\end{gathered}
$$

They are dual in the sense that $\Pi(\varphi)=1-N(\neg \varphi)$ for every proposition $\varphi$. From a logical point of view, possibilistic logic can be seen as a sort of graded extension of the non-nested fragment of the well-known modal logic of belief KD45.

When we go beyond the classical framework of Boolean algebras of events to generalized algebras of many-valued events, one has to come up with appropriate extensions of the notion of necessity and possibility measures for many-valued events, as explored in [DGM11]. A natural generalization is to consider $\Omega$ as the set of propositional interpretations of some many-valued calculi defined by a t-norm $\odot$ and its residuum $\Rightarrow$, in particular when $\odot$ is the product t-norm. Then, a possibility distribution $\pi: \Omega \rightarrow[0,1]$ induces the following generalized possibility and necessity measures over many-valued propositions:

$$
\begin{aligned}
& \Pi(\varphi):=\sup \{\pi(w) \odot w(\varphi) \mid w \in \Omega\} \\
& \mathrm{N}(\varphi):=\inf \{\pi(w) \Rightarrow w(\varphi) \mid w \in \Omega\} .
\end{aligned}
$$

Actually, these definitions agree with the ones commonly used in many-valued modal logics with Kripke semantics based on frames $(W, R)$ with $R$ being a [0,1]-valued binary relations $R: W \times W \rightarrow$ $[0,1]$ (see for example [BEGR11]), in the particular case where the many-valued accessibility relations $R$ are of the form $R\left(w, w^{\prime}\right)=\pi\left(w^{\prime}\right)$, for some possibility distributions $\pi: W \rightarrow[0,1]$. In the frame of this paper, we generalize this possibilistic semantics by replacing the unit real interval $[0,1]$ by an arbitrary product algebra.

The set of formulas over the language of the product logic $\Pi^{\infty}$ extended with two unary operators, $\mathrm{N}$ and $\Pi$, will be denoted by $F m_{\mathrm{Pos}_{\Pi}}$ and we will refer to them as possibilistic formulas.

Definition 6.6. Let $\boldsymbol{A}$ be a $\Pi^{\infty}$-algebra. An $\boldsymbol{A}$-possibilistic model is a structure $\langle W, \pi, e\rangle$ such that

- $W$ is a non-empty set of worlds;

- $\pi: W \rightarrow \boldsymbol{A}$ is an $\boldsymbol{A}$-valued possibility distribution;

- $e: W \times \mathcal{V} \rightarrow \boldsymbol{A}$ is an evaluation of variables in each world. It extends to all the formulas by interpreting the propositional connectives by the corresponding operations of $\boldsymbol{A}$, and the possibilistic operations by

$$
e(v, \Pi \varphi)=\sup _{w \in W}(\pi(w) \odot e(w, \varphi)\} \quad e(v, \mathrm{~N} \varphi)=\inf _{w \in W}(\pi(w) \rightarrow e(w, \varphi)\} .
$$

Again, if these latter two values exist for any formula in any world, the model is called safe. The class of safe Possibilistic models over product algebras will be denoted by $\operatorname{Pos}_{\Pi}$. For $\Gamma \cup\{\varphi\} \subseteq$ $F m_{\text {Pos }_{\Pi}}$, we will write $\Gamma \models \operatorname{Pos}_{\Pi} \varphi$ whenever for any $\mathfrak{M} \in \operatorname{Pos}_{\Pi}$ and any $w \in W, e(w,[\Gamma]) \subseteq\{1\}$ implies that $e(w, \varphi)=1$. 
Notation: For the sake of uniformity with the notation used in existing literature, from now on we will denote $\mathrm{PK}^{\{T, 5\}}$ by PS5 (i.e., the set of reflexive and euclidean safe product models), and $\mathbf{K} \Pi^{\infty\{\mathrm{T}, 5\}}$ by $\mathbf{S 5}_{\Pi}$, the logic which is strongly complete with respect to that class of models.

In $\left[\mathrm{HHE}^{+} 94\right]$, it is shown how a possibilistic modal logic over a finitely-valued Łukasiewicz logic can be embedded in a $S 5$-like extension (with axioms $(\mathrm{K}),(\mathrm{T})$ and (5)) over a language extended with a new propositional variable, playing the special role of the possibility distribution. We follow the same idea here.

We let $\star$ to be the translation from $F m_{\mathrm{Pos}_{\Pi}}$ (built from a set $\mathcal{V}$ of propositional variables) to the set of modal ${ }^{13}$ formulas built from $\mathcal{V} \cup\{p\}$, with $\{p\}$ a new fresh variable not in $\mathcal{V}$, defined as:

$$
\begin{aligned}
\bar{c}^{\star}:=\bar{c}, \quad x^{\star}:=x \text { for } x \in \mathcal{V} & (\Delta \varphi)^{\star}:=\Delta \varphi^{\star} \\
(\varphi \& \psi)^{\star}:=\varphi^{\star} \& \psi^{\star} & (\mathrm{N} \varphi)^{\star}:=\square\left(p \rightarrow \varphi^{\star}\right) \\
(\varphi \rightarrow \psi)^{\star}:=\varphi^{\star} \rightarrow \psi^{\star} & (\Pi \varphi)^{\star}:=\diamond\left(p \& \varphi^{\star}\right) .
\end{aligned}
$$

Theorem 6.7. For any set of possibilistic formulas $\Gamma \cup\{\varphi\} \subseteq F m_{\mathrm{Pos}_{\Pi}}$ it holds that

$$
\Gamma \models \operatorname{Pos}_{\Pi} \varphi \text { iff } \Gamma^{\star} \vdash_{\mathbf{S 5}_{\Pi}} \varphi^{\star} \text {. }
$$

Proof. First, given $\mathfrak{M}=\langle W, \pi, e\rangle \in \operatorname{Pos}_{\Pi}$, a model $\mathfrak{M}^{\prime}=\left\langle W^{\prime}, R^{\prime}, e^{\prime}\right\rangle \in$ PS5 can be defined by taking $W^{\prime}=W, R^{\prime}=W \times W, e^{\prime}(v, x)=e(v, x)$ for all $x \in \mathcal{V}$ and $e^{\prime}(v, p)=\pi(v)$. By the definition of the evaluation of the modal formulas in a possibilistic model, and the behaviour of the translation $\star$ over them, it is immediate to see, by induction on the length of the formula, that the evaluations coincide, that is, $e(v, \psi)=e^{\prime}\left(v, \psi^{\star}\right)$ for any $\psi \in F m_{\mathrm{Pos}_{\Pi}}$. Take for instance the possibilistic formula $\mathbf{N} \chi$. By definition, $e(v, \mathbf{N} \chi)=\inf _{w \in W}(\pi(w) \rightarrow e(w, \chi)\}=\inf _{w \in W}\left\{e^{\prime}(w, p) \rightarrow e(w, \chi)\right\}$. By the induction hypothesis, this is equal to $\inf _{w \in W}\left\{e^{\prime}(w, p) \rightarrow e^{\prime}\left(w, \chi^{\star}\right)\right\}$, and thus, to $\inf _{w \in W}\left\{e^{\prime}(w, p \rightarrow\right.$ $\left.\left.\chi^{\star}\right)\right\}=e^{\prime}\left(v, \square\left(p \rightarrow \chi^{\star}\right)\right)=e^{\prime}\left(v,(\mathrm{~N} \chi)^{\star}\right)$.

Conversely, given a model $\mathfrak{M} \in \mathrm{PS} 5$, define $\mathfrak{M}^{\prime} \in \operatorname{Pos}_{\Pi}$ by letting $W^{\prime}=W, \pi(v)=e(v, p)$ and $e^{\prime}(v, x)=e(v, x)$ for all $x \in \mathcal{V}$ and $v \in W$. Then it is also easy to check, again by induction, that $e\left(v, \psi^{\star}\right)=e^{\prime}(v, \psi)$ for all $\psi \in F m_{\text {Pos }_{\Pi}}$ and $v \in W$. Similarly as before, consider the case where $\psi=\mathrm{N} \chi$, that is, $\psi^{\star}=\square\left(p \rightarrow \chi^{\star}\right)$. Then, by definition, $e\left(v, \square\left(p \rightarrow \chi^{\star}\right)=\inf _{w \in W}\left\{e\left(w, p \rightarrow \chi^{\star}\right)\right\}=\right.$ $\inf _{w \in W}\left\{e(w, p) \rightarrow e\left(w, \chi^{\star}\right)\right\}=\inf _{w \in W}\left\{\pi(w) \rightarrow e\left(w, \chi^{\star}\right)\right\}$. By the induction hypothesis, this is equal to $\inf _{w \in W}\left\{\pi(w) \rightarrow e^{\prime}(w, \chi)\right\}$ which is equal, by definition, to $e^{\prime}(v, \mathrm{~N} \chi)$.

At this point, one can wonder if this logic indeed behaves as expected as a logic for reasoning about graded possibilities and necessities. The main discrepancy is the behaviour of the necessity operator over negated formulas. Indeed, it is straightforward to check that, in any possibilistic model $\mathfrak{M}=(W, \pi, e) \in \operatorname{Pos}_{\Pi}$, we have that for any $v \in W$ :

$$
e(v, \mathrm{~N} \neg \varphi)= \begin{cases}1, & \text { if, for all } w \in W, \text { either } \pi(w)=0 \text { or } e(w, \varphi)=0 \\ 0, & \text { otherwise }\end{cases}
$$

This means that $\mathrm{N}$ behaves as a Boolean operator over the negated events, which is rather unintuitive since, in contrast to classical Possibilistic logic, it means that uncertainty (in terms of necessity measures) on negated formulas (even if they are Boolean themselves) cannot be graded, so a negated formula can only fully believed or fully disbelieved. Therefore, it makes full sense to consider a language where it is possible to build more expressive formulas to reason about negated events. A natural solution is to expand the language with a new negation connective $\sim$ and endowing it with a behaviour of an involutive negation (that within the standard product algebra is simply given by $\left.\sim[0,1]_{\Pi} a=1-a\right)$.

At a propositional level, there are several works about the extension of product logic with an involutive negation $\sim([\mathrm{CKMN10}],[\mathrm{CKMN06}],[\mathrm{EGHN00]})$, and one of the first remarks is that the

\footnotetext{
${ }^{13}$ With usual $\square$ and $\diamond$ operators.
} 
Baaz-Monteiro operator $\Delta$ is in fact definable from the two negations: $\Delta \varphi=\neg \sim \varphi$. Moreover, with the involutive negation it is not necessary to add rule $R_{\Delta}$, since it turns to be derivable.

So we define $\Pi_{\sim}^{\infty}$ to be the following axiomatization for a product logic with rational constants and an involutive negation:

- axioms of $\Pi^{c}$, i.e., Product Logic axioms and rules plus the following ones:

$$
\begin{gathered}
\left(A_{c} 1\right) \bar{c} \& \bar{d} \leftrightarrow \overline{c \cdot d} \\
\left(A_{c} 2\right)(\bar{c} \rightarrow \bar{d}) \leftrightarrow \overline{c \Rightarrow_{\Pi} d} \\
\left(N_{\neg \sim}\right) \frac{\varphi}{\Delta \varphi} \\
\left(\mathrm{R}_{\rightarrow}\right) \frac{\{\varphi \rightarrow \bar{c}\}_{c \in(0,1)_{\mathbb{Q}}}}{\neg \varphi} \\
\text { - } \neg \varphi \rightarrow \sim \varphi \\
\text { - } \sim \varphi \rightarrow \varphi \\
\text { - } \Delta(\varphi \rightarrow \psi) \rightarrow(\sim \psi \rightarrow \sim \varphi) \\
\text { - } \sim \bar{c} \leftrightarrow \overline{1-c}
\end{gathered}
$$

It was remarked in [Cin04] that this logic coincides with the so called Eukasiewicz Product Logic with rational truth constants $\mathrm{L} \Pi \frac{1}{2}$ [EGM01]. This is so because the definition of the $\sim$ operator over the rational constants, which are dense in $[0,1]$, uniquely determines that in the whole real interval $[0,1] \sim$ has to be exactly the standard Eukasiewicz negation $1-x$. This allows all the other Łukasiewicz connectives (in particular the Eukasiewicz strong conjunction $\&_{€}$ and implication $\rightarrow_{€}$ ), as well as all rational constants besides $\frac{\overline{1}}{2}$, to be definable in $\Pi_{\sim}^{\infty}$.

The axiomatization of a modal logic (with modal operators $\square$ and $\diamond$ ), strongly complete with respect to the class of (crisp) Kripke models over product algebras with an involutive negation, is naturally obtained by extending $\Pi_{\sim}^{\infty}$ with the expected axioms $(\mathrm{K}),\left(\mathrm{A}_{\square} 1\right),\left(\mathrm{A}_{\diamond} 1\right)$ and $\left(\mathrm{A}_{\square} 2\right)$. We will denote this logic by $\mathbf{K} \Pi_{\sim}^{\infty}$. It is simple to check that it is complete with respect to the class of Kripke structures evaluated over product algebras with rational constants and with an involutive negation. Note that, considering a negated formula of the kind $\sim \varphi$, i.e. with the involutive negation instead of the usual one, the evaluation of its necessity in a certain possibilistic model $\mathfrak{M}=(W, \pi, e)$ over the canonical standard product algebra expanded with the involutive negation $1-x$ is as follows:

$$
e(v, \mathrm{~N} \sim \varphi)=\inf _{w \in W}\left(\pi(x) \Rightarrow_{\Pi} 1-e(w, \varphi)\right),
$$

which is no longer $\{0,1\}$-valued. Moreover, one could consider as well another necessity operator $\mathbf{N}^{\prime}$, definable in $\mathbf{K} \Pi_{\sim}^{\infty}$, resulting from dualizing the possibility operator $\Pi$ with the involutive negation $\sim$, namely one can define $\mathrm{N}^{\prime} \varphi:=\sim \Pi \sim \varphi$. The semantics of this operator (over the above model over $[0,1])$ is given by the following expression:

$$
e\left(v, \mathrm{~N}^{\prime} \varphi\right)=\inf _{w \in W}(1-\pi(x)+\pi(x) \cdot e(w, \varphi))
$$

The semantics of the pair of operators $\Pi$ and $\mathrm{N}^{\prime}$ are indeed proper extensions of the original possibility and necessity measures for classical propositions used in Dubois and Prade's Possibilistic logic, while the one for $\mathrm{N}$ is not, as shown above.

Following an analogous approach to the one used for proving completeness of $\mathbf{K} \Pi^{\infty}$ by means of a canonical model construction (Theorem 4.11), we can check completeness of $\mathbf{K} \Pi_{\sim}^{\infty}$ as well. Indeed, the canonical model of $\mathbf{K} \Pi^{\infty}$ can be defined in the natural way: $\mathfrak{M}_{\mathfrak{c}}^{\sim}=\left\langle W_{c}^{\sim}, R_{\mathfrak{c}}^{\sim}, e_{\mathfrak{c}}^{\sim}\right\rangle$ where $W_{c}^{\sim}:=\left\{h \in \operatorname{Hom}\left(\left(F m_{\mathbf{K} \Pi_{\sim}^{\infty}}\right)^{*},[0,1]_{\Pi_{\sim}}\right): h\left(\left(T h_{\mathbf{K}_{\Pi_{\sim}}}\right)^{*}\right) \subseteq\{1\}\right\}, R_{\mathfrak{c}}^{\sim} v w$ iff for any $\psi \in F m_{\mathbf{K} \Pi_{\sim}^{\infty}}$ $v\left(\theta_{\square}\right)=1$ implies $w\left(\theta^{*}\right)=1$, and $e_{\mathfrak{c}}^{\sim}(w, x):=w(x)$ for every $x \in \mathcal{V}$. 
Notice that, since $\mathbf{K} \Pi_{\sim}^{\infty}$ extends Eukasiewicz logic, it follows that the modal operators $\square$ and $\diamond$ are now fully interdefinable, i.e., $\diamond \varphi$ is equivalent to $\sim \square \sim \varphi$, and $\square \varphi$ equivalent to $\sim \diamond \sim \varphi$.

It is now possible to follow the same reasoning as the one done for Theorem 6.7 to prove a completeness result for the possibilistic (local) modal logic $\operatorname{Pos}_{\Pi \sim}$ by embedding it into the logic $\mathbf{S} \mathbf{5}_{\Pi \sim}$ (the extension of $\mathbf{S} \mathbf{5}_{\Pi}$ with an involutive negation in the natural way). It is just necessary to observe that, as expected, $(\sim \varphi)^{\star}=\sim \varphi^{\star}$.

Theorem 6.8. For any set of possibilistic formulas $\Gamma \cup\{\varphi\} \subseteq F m_{\mathrm{Pos}_{\Pi \sim}}$

$$
\Gamma \models \operatorname{Pos}_{\Pi \sim} \varphi \text { iff } \Gamma^{\star} \vdash_{\mathbf{S 5}_{\Pi \sim}} \varphi^{\star} .
$$

As a final observation, we show what are the translations in the language of $\mathbf{S} \mathbf{5}_{\Pi \sim}$ of the dual operators of $\mathrm{N}_{\Pi}$ and $\Pi_{\Pi}$ through the involutive negation $\sim$.

Lemma 6.9. The following are theorems in $\mathbf{S} \mathbf{5}_{\Pi_{\sim}}$ :

(1) $\left(\Pi^{\prime} \varphi\right)^{\star} \leftrightarrow \diamond\left(\neg \neg p \wedge\left(p \rightarrow p \&_{€} \varphi\right)\right)$;

(2) $\left(\mathrm{N}^{\prime} \varphi\right)^{\star} \leftrightarrow \square\left(p \rightarrow_{E} p \& \varphi\right)$.

where $\Pi^{\prime} \varphi$ is an abbreviation for $\sim \mathrm{N} \sim \varphi$ (recall that $\mathrm{N}^{\prime} \varphi$ stands for $\sim \Pi \sim \varphi$ ).

Proof. (1) By completeness, we have that on any Kripke $[0,1]_{\Pi \sim}-$ model $\mathfrak{M}=(W, \pi, e)$ with a reflexive and euclidean accessibility relation $R$, for any $w \in W$, we have $e\left(w,(\sim N \sim \varphi)^{\star}\right)=$ $1-e\left(w, \square\left(p \rightarrow \sim \varphi^{\star}\right)\right)=1-\inf \left\{e\left(v, p \rightarrow \sim \varphi^{\star}\right): v \in W\right\}=\sup \left\{1-e\left(v, p \rightarrow \sim \varphi^{\star}\right): v \in W\right\}$. We can prove by cases (depending on the value of $p$ in the world) that for each $v \in W$, $1-e\left(v, p \rightarrow \sim \varphi^{\star}\right)=e\left(v, \neg \neg p \wedge\left(p \rightarrow p \&_{£} \varphi\right)\right)$.

- For $v \in W$ such that $e(v, p)=0$, it holds that $1-e\left(v, p \rightarrow \sim \varphi^{\star}\right)=0$, which is equal to $e(v, \neg \neg p)$.

- For $v \in W$ such that $e(v, p)>0$, note that $e(v, \neg \neg p)=1$, so it does not affect the value of the conjunction. Therefore it remains to be proved that $1-e\left(v, p \rightarrow \sim \varphi^{\star}\right)=$ $e\left(v, p \rightarrow p \&_{\mathrm{E}} \varphi^{\star}\right)$. We consider two cases:

$\left.{ }^{*}\right)$ If $e(v, p) \leqslant e\left(v, \sim \varphi^{\star}\right)$ then $1-e\left(v, p \rightarrow \sim \varphi^{\star}\right)=0$. Note that $e(v, p) \leqslant e\left(v, \sim \varphi^{\star}\right)$ whenever $e(v, p)-1+e\left(v, \varphi^{\star}\right) \leqslant 0$, or equivalently $e\left(v, p \&_{\mathrm{E}} \varphi^{\star}\right)=0$. Given that $e(v, p)>0$, we have that $e\left(v, p \rightarrow p \&_{\mathrm{E}} \varphi^{\star}\right)=0$ as well.

$\left(^{*}\right.$ If $e(v, p)>e\left(v, \sim \varphi^{\star}\right)$, by the definition of the product implication on $[0,1]$ we can directly write $1-e\left(v, p \rightarrow \sim \varphi^{\star}\right)=\frac{e(v, p)-1+e\left(v, \varphi^{\star}\right)}{e(v, p)}=\frac{e\left(v, p \&_{\mathrm{E}} \varphi^{\star}\right)}{e(v, p)}$ and, since $e\left(v, p \&_{\mathrm{E}} \chi\right) \leqslant$ $e(v, p)$ for any formula $\chi$, this equals to $e\left(v, p \rightarrow p \&_{\mathrm{E}} \varphi^{\star}\right)$.

(2) Similarly, we have that $e\left(w,(\sim \Pi \sim \varphi)^{\star}\right)=1-e\left(w, \diamond\left(p \& \sim \varphi^{\star}\right)\right)=1-\sup \left\{e\left(v, p \& \sim \varphi^{\star}\right): v \in\right.$ $W\}=\inf \left\{1-e\left(v, p \& \sim \varphi^{\star}\right): v \in W\right\}=\inf \left\{1-\left(e(v, p) \cdot\left(1-e\left(v, \varphi^{\star}\right)\right): v \in W\right\}=\inf \{1-\right.$ $\left.e(v, p)+e(v, p) \cdot e\left(v, \varphi^{\star}\right): v \in W\right\}=\inf \left\{e(v, p) \rightarrow_{\mathrm{L}} e\left(v, p \& \varphi^{\star}\right)\right\}\left(\right.$ since $e(v, p) \geqslant e\left(v, p \& \varphi^{\star}\right)$ in any case).

\section{CONCLUding REMARKS}

In this paper we have mainly been concerned with the study and axiomatization of modal extensions of the Product fuzzy logic with semantics given by Kripke frames with crisp accessibility relations and evaluations over the canonical standard product algebra. To do this, we have first developed an infinitary product logic $\Pi^{\infty}$ with rational constants and with Monteiro-Baaz's Delta operator (both from an axiomatic and semantical points of view), with the objective of providing a solid basis to build modal extension, that is the core of this paper. Then, we have provided axiomatizations for the minimal local and global modal logics extending $\Pi^{\infty}$ and proved completeness with respect to different classes of Kripke frames evaluated over product algebras. In order to deepen the analysis of these logics, we have also developed their algebraic semantics and studied its 
relationship with the Kripkean semantics. Finally, we have also considered some usual axiomatic extensions of modal logics in our particular framework.

The need of resorting to the use of rational constants together with two infinitary inference rules to handle them, as well the Monteiro-Baaz Delta operator, has been motivated by our aim to get complete modal axiomatizations with respect to corresponding classes of Product Kripke models. We admit this approach may be felt as not very elegant, but we have not been able to find a better alternative. Note that also in [BEGR11], an intensive use of constants in the language is made for the analysis of modal logics over finite MTL-algebras.

There are a number of interesting open problems that we plan to address in future works. In particular, one natural question is the study of a modal extension of product fuzzy logic arising from Kripke models where the accessibility relation is fuzzy as well, as it is the case e.g. in [CR10, CR15, BEGR11]. In such a case, axiom $\mathrm{K}$ is no longer sound, and this may make the problem more complex. Another pending question is to study decidability issues of the logics defined in this paper. Note that in [Háj10] the arithmetical complexity of a modal extension of product logic (without truth-constants and $\Delta$ operator) with an S5 semantics was also left open. There are however a few available results in the literature in similar settings: in [CEB10] the authors show that checking whether a formula is positive-satisfiable or 1-valid in a modal product logic (without truth-constants and Delta) is decidable. However the techniques used in that paper cannot be applied here because of the presence of truth-constants and the $\Delta$ operator. Also, in [CMRR13] it is shown that Gödel modal logics are decidable by defining an alternative new semantics for which the finite model property holds.

On the other hand, one can realise that many of the results obtained in this paper do not rely on characteristic properties of Product logic itself but on weaker assumptions. With this in mind, it seems that the step to understand modal extensions of weaker fuzzy logics (for instance, classes of MTL logics) could be not too far. This problem is part of ongoing work.

Acknowlegdements. The authors wish to thank the anonymous reviewers for their valuable comments and suggestions that have significantly improved the paper. They also thank Tommaso Moraschini for helpful comments on Section 5. They also acknowledge support of the Spanish projects EdeTRI (TIN2012-39348- C02-01) and 2014 SGR 118. Amanda Vidal was supported by a CSIC grant JAE Predoc.

\section{REFERENCES}

$\left[\mathrm{BEF}^{+} 09\right]$ F. Bou, F. Esteva, J. M. Font, A. Gil, L. Godo, A. Torrens, and V. Verdú. Logics preserving degrees of truth from varieties of residuated lattices. Journal of Logic and Computation, 19(6):1031-1069, 2009.

[BEGR11] F. Bou, F. Esteva, L. Godo, and R. Rodríguez. On the minimum many-valued modal logic over a finite residuated lattice. Journal of Logic and Computation, 21(5):739-790, 2011.

[BP86] W. J. Blok and D. Pigozzi. Protoalgebraic logics. Studia Logica, 45:337-369, 1986.

[BP89] W. J. Blok and D. Pigozzi. Algebraizable logics, volume 396 of Mem. Amer. Math. Soc. A.M.S., Providence, January 1989.

[CE11] M. Cerami and F. Esteva. Strict core fuzzy logics and quasi-witnessed models. Archive for Mathematical Logic, 50(5):625-641, 2011.

[CEB10] M. Cerami, F. Esteva, and F. Bou. Decidability of a description logic over infinite-valued product logic. In F. Lin, U. Sattler, and M. Truszczynski, editors, Principles of Knowledge Representation and Reasoning: Proceedings of the Twelfth International Conference, KR 2010, Toronto, Ontario, Canada, May 9-13, 2010, pages 203-213. AAAI Press, 2010.

[Cin01] P. Cintula. About axiomatic systems of product fuzzy logic. Soft Computing, 5(3):243-244, 2001.

[Cin04] P. Cintula. From fuzzy logic to fuzzy mathematics. Ph. D. thesis, Czech Technical University in Prague, 2004.

[Cin14] P. Cintula. A note on axiomatizations of pavelka-style complete fuzzy logics. Fuzzy Sets and Systems, page In Press, 2014.

[CKMN06] P. Cintula, E. P. Klement, R. Mesiar, and M. Navara. Residuated logics based on strict triangular norms with an involutive negation. Mathematical Logic Quarterly, 52(3):269-282, 2006. 
[CKMn10] P. Cintula, E. Peter Klement, R. Mesiar, and M. Navara. Fuzzy logics with an additional involutive negation. Fuzzy Sets and Systems, 161(3):390-411, 2010.

[CMRR13] X. Caicedo, G. Metcalfe, R. Rodríguez, and J. Rogger. A finite model property for Gödel modal logics. In Leonid Libkin, Ulrich Kohlenbach, and Ruy de Queiroz, editors, WoLLIC, Lecture Notes in Computer Science 8071, pages 226-237, 2013.

[CR10] X. Caicedo and R. Oscar Rodríguez. Standard Gödel modal logics. Studia Logica, 94(2):189-214, 2010.

[CR15] Xavier Caicedo and Ricardo Oscar Rodríguez. Bi-modal gödel logic over [0, 1]-valued kripke frames. J. Log. Comput., 25(1):37-55, 2015.

[CT00] R. Cignoli and A. Torrens. An algebraic analysis of product logic. Multiple-valued logic, 5:45-65, 2000.

[CZ97] A. Chagrov and M. Zakharyaschev. Modal Logic, volume 35 of Oxford Logic Guides. Oxford University Press, 1997.

[Cze01] J. Czelakowski. Protoalgebraic logics, volume 10 of Trends in Logic-Studia Logica Library. Kluwer Academic Publishers, Dordrecht, 2001.

[DGM11] P. Dellunde, L. Godo, and E. Marchioni. Extending possibilistic logic over Gödel logic. International Journal of Approximate Reasoning, 52(1):63-75, 2011.

[DLP94] D. Dubois, J. Lang, and H. Prade. Possibilistic logic. In Handbook of logic in artificial intelligence and logic programming, Vol. 3, Oxford Sci. Publ., pages 439-513. Oxford Univ. Press, New York, 1994.

[DP04] D. Dubois and H. Prade. Possibilistic logic: a retrospective and prospective view. Fuzzy Sets and Systems, 144(1):3-23, 2004.

[EGHN00] F. Esteva, L. Godo, P. Hájek, and M. Navara. Residuated fuzzy logics with an involutive negation. Archive for Mathematical Logic, 39(2):103-124, 2000.

[EGM01] F. Esteva, L. Godo, and F. Montagna. The $L \Pi$ and $L \Pi \frac{1}{2}$ logics: two complete fuzzy systems joining Łukasiewicz and Product Logics. Archive for Mathematical Logic, 40(1):39-67, 2001.

[FJP03] J. M. Font, R. Jansana, and D. Pigozzi. A survey on abstract algebraic logic. Studia Logica, Special Issue on Abstract Algebraic Logic, Part II, 74(1-2):13-97, 2003. With an "Update" in 91 (2009), 125-130.

[Háj98] P. Hájek. Metamathematics of fuzzy logic, volume 4 of Trends in Logic-Studia Logica Library. Kluwer Academic Publishers, Dordrecht, 1998.

[Háj10] P. Hájek. On fuzzy modal logics S5(C). Fuzzy Sets and Systems, 161(18):2389-2396, 2010.

[HGE96] P. Hájek, L. Godo, and F. Esteva. A complete many-valued logic with product-conjunction. Archive for Mathematical Logic, 35:191-208, 1996.

$\left[\mathrm{HHE}^{+} 94\right]$ P. Hájek, D. Harmancová, F. Esteva, P. Garcia, and L. Godo. On modal logics for qualitative possibility in a fuzzy setting. In R. López de Mántaras and D. Poole, editors, Proceedings of the 10th Annual Conference on Uncertainty in Artificial Intelligence (UAI-94), pages 278-285, San Francisco, CA, 1994. Morgan Kaufmann.

[HT13] G. Hansoul and B. Teheux. Extending łukasiewicz logics with a modality: Algebraic approach to relational semantics. Studia Logica, 101(3):505-545, 2013.

[Jen05] S. Jenei. On the determination of left-continuous t-norms and continuous archimedean t-norms on some segments. Aequationes Mathematicae, 70:177-188, 2005.

[Mal86] J. Malinowski. Modal equivalential logics. Journal of Non-Classical Logic, 3:13-35, 1986.

[MNH06] F. Montagna, C. Noguera, and R. Horčík. On weakly cancellative fuzzy logics. Journal of Logic and Computation, 16(4):423-450, 2006.

[Mon06] F. Montagna. Notes on strong completeness in Łukasiewicz, product and BL logics and in their first-order extensions. In Algebraic and Proof-theoretic Aspects of Non-classical Logics, pages 247-274, 2006.

[Ost88] P. Ostermann. Many-valued modal propositional calculi. Zeitschrift für Mathematische Logik und Grundlagen der Mathematik, 34(4):343-354, 1988.

[SCE $\left.{ }^{+} 06\right]$ P. Savický, R. Cignoli, F. Esteva, L. Godo, and C. Noguera. On product logic with truth-constants. Journal of Logic and Computation, 16(2):205-225, 2006.

[VEG14] Amanda Vidal, Francesc Esteva, and Lluís Godo. About standard completeness of product logic. In Proc. of the XVII Congreso Español sobre Tecnologías y Lógica Fuzzy (ESTYLF 2014), pages 423-428, Zaragoza, 05/02/2014 2014.

\section{ANNEX}

[Proof of Lemma 3.5] Let $\boldsymbol{A}$ be a $\Pi^{\infty}$-algebra and $F$ a $\Pi^{\infty}$-filter over $\boldsymbol{A}$. Then $F$ is the intersection of all the linear $\Pi^{\infty}$-filters over $\boldsymbol{A}$ that contain it.

Proof. Although, strictly speaking, the claim of lemma is not a particular case of more general results that can be found in the literature (the logic is infinitary and its corresponding class of 
algebras is not a variety but a generalised quasi-variety), all the needed steps in this proof are in fact natural extensions of the ones in the literature (in our case we mainly relyi on [Cze01]).

Being $\mathbb{P}^{\infty}$ a generalized quasi-variety, we know it is closed under subdirect products, $\mathbb{P}_{S D}$. Remember that an algebra $\boldsymbol{A}$ is a subdirect product of a family of algebras $\left\{\boldsymbol{A}_{i}\right\}_{i \in I}$ whenever there is an embedding $\sigma$ from $\mathbf{A}$ into $\Pi_{i \in I} A_{i}$ such that $\pi_{i} \circ \sigma$ is surjective. It is important to recall that given $\boldsymbol{A} \in \mathbb{P}^{\infty}$ and $\theta \cup\left\{\theta_{i}\right\}_{i \in I} \subseteq C_{o_{\mathbb{P}} \infty} \mathbf{A}, \boldsymbol{A} / \theta$ is subdirect product of $\left\{\boldsymbol{A} / \theta_{i}: i \in I\right\}$ if and only if $\theta=\bigcap_{i \in I} \theta_{i}$.

Let $\left(\mathbb{P}^{\infty}\right)_{R S I}$ be the class of subdirectly irreducible algebras relative to $\mathbb{P}^{\infty}$, i.e., if $\boldsymbol{A} \in \mathbb{P}^{\infty}$ is such that $\boldsymbol{A} \in \mathbb{P}_{S D}\left\{\boldsymbol{B}_{i}\right\}_{i \in I}$ with $\boldsymbol{B}_{i} \in \mathbb{P}^{\infty}$, then $\boldsymbol{A} \cong \mathbf{B}_{\mathbf{i}}$ for some $i \in I$. Generalizing Birkoff representation theorem, $\mathbb{P}^{\infty}=\mathbb{P}_{S D}\left(\left(\mathbb{P}^{\infty}\right)_{R S I}\right)$. Then for any $\boldsymbol{A} \in \mathbb{P}^{\infty}$ and $\theta \in C o_{\mathbb{P}} \boldsymbol{A}, \theta=\bigcap\{\Phi \in$ $C_{\mathbb{P} \infty} \boldsymbol{A}: \boldsymbol{A} / \Phi \in\left(\mathbb{P}^{\infty}\right)_{R S I}$ and $\left.\theta \subseteq \Phi\right\}$. Using properties of the congruences, it is easy to prove that $\boldsymbol{A} / \Phi \in\left(\mathbb{P}^{\infty}\right)_{R S I}$ if and only if $\Phi$ is $\bigcap$-irreducible in $C_{\mathbb{P} \infty} \boldsymbol{A}$, i.e., if $\Phi=\bigcap_{i \in I}\left\{\Phi_{i}\right\}$, then there is $i \in I$

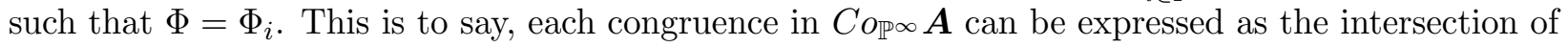
all the $\bigcap$-irreducible congruences in $C_{\mathbb{P} \infty} \boldsymbol{A}$ that contain it. Due to the algebraizability of $\Pi^{\infty}$, this is equivalent to have that each $\Pi^{\infty}$-filter $F$ of $\boldsymbol{A}$ is the intersection of all the $\bigcap$-irreducible filters

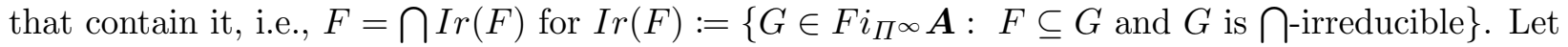
$\operatorname{Ir}_{\omega}(F):=\left\{G \in F i_{\Pi \infty \boldsymbol{A}}: F \subseteq G\right.$ and $G$ is finitely $\bigcap$-irreducible $\}$. It is clear that $\operatorname{Ir}(F) \subseteq \operatorname{Ir}_{\omega}(F)$, and thus, $\bigcap \operatorname{Ir}_{\omega}(F) \subseteq \bigcap \operatorname{Ir}(F)$. On the other hand, since $F \subseteq G$ for all $G \in \operatorname{Ir}_{\omega}(F), F \subseteq \bigcap \operatorname{Ir}_{\omega}(F)$, we have that $F \subseteq \bigcap \operatorname{Ir}_{\omega}(F) \subseteq \bigcap \operatorname{Ir}(F)=F$, so $F=\bigcap \operatorname{Ir}_{\omega}(F)$.

We conclude the proof checking that the finitely $\bigcap$-irreducible filters are exactly the linear ones. The right to left direction is immediate. The other direction can be proved in an analogous way to [Cze01, Cor. 2.5.4.]. There are only two main differences to take into account. First, the one of defining the semantical proof (of an element $a$ belonging to the filter generated by a set of elements $X)$ in an infinitary way, following the syntactical definition of proof from 3.3. And second, just observing that both the two new infinitary rules are closed under $\vee\left(\right.$ see $\left(R^{\prime}{ }_{\Delta}\right)$ and $\left(R_{\rightarrow}^{\prime}\right)$ in Section 3.

[Proof of Lemma 3.9] Let $\boldsymbol{A}$ be a $\Pi^{\infty}$-algebra linearly ordered. Then $\boldsymbol{A}$ can be embedded in the canonical standard product algebra $[0,1]_{\Pi}$ by a unique complete embedding.

Proof. In [CT00] it is shown that there exist two functors $\mathfrak{G}: P L_{c} \leftrightarrow L G: \mathfrak{B}$ that induce a natural equivalence between the full subcategory of product algebras satisfying a certain condition and the category of the lattice-ordered abelian groups. For the particular case of linearly ordered product algebras (that satisfy the mentioned condition) and linearly ordered abelian groups (which by cardinality reasons are isomorphic to the additive group of the real numbers, $\mathbb{R}_{+}=\langle\mathbb{R},+,-, 0\rangle$ ), this equivalence is as follows. Given a linearly ordered $\Pi$-algebra $\boldsymbol{A}=\left\langle A, \odot, \rightarrow, 0^{\boldsymbol{A}}, 1^{\boldsymbol{A}}\right\rangle, \mathfrak{G}(\boldsymbol{A})$ is defined as the linearly ordered abelian group whose universe is $\left\{a_{+}: a \in A \backslash\{\perp, 1\}\right\} \cup\left\{a_{-}: a \in\right.$ $A \backslash\{\perp, 1\}\} \cup\{0\}$, where $\mathbf{O}$ is the neutral element (that coincides with $1_{+}^{\boldsymbol{A}}$ and $1_{-}^{\boldsymbol{A}}$ ), the order is given by $a_{-}<_{\mathfrak{G}(A)} b_{-}<_{\mathfrak{G}(A)} 0<_{\mathfrak{G}(A)} b_{+}<_{\mathfrak{G}(A)} a_{+}$for $0<_{\boldsymbol{A}} a<_{\boldsymbol{A}} b \in A$, and the group operations (addition and inverse) + and - are defined by:

$$
\begin{aligned}
a_{+}+b_{+} & =(a \odot b)_{+} \\
a_{-}+b_{-} & =(a \odot b)_{-} \\
a_{+}+b_{-} & = \begin{cases}\mathbf{0} & \text { if } a=\boldsymbol{A}_{\boldsymbol{A}} b \\
(b \rightarrow a)_{-} & \text {if } a<_{\boldsymbol{A}} b \\
(a \rightarrow b)_{+} & \text {if } b<_{\boldsymbol{A}} a .\end{cases} \\
-a_{+} & =a_{-} \\
-a_{-} & =a_{+}
\end{aligned}
$$


Conversely, given a linearly ordered abelian group $\boldsymbol{G}=\langle G,+,-, \leqslant, 0\rangle$, we define $\mathfrak{B}(G)$ as the product chain with the universe $\{g \in G: g \leqslant 0\} \cup\{-\infty\}$, with the order $\leqslant_{\mathfrak{B}(G)}$ being the order of $G$ extended by letting $-\infty \leqslant_{\mathfrak{B}(G)} g$ for any $g \in G$, and with operations:

$$
a \odot b=\left\{\begin{array}{ll}
a+b & \text { if } a, b \in G \\
-\infty & \text { otherwise }
\end{array} \quad a \rightarrow b= \begin{cases}0 & \text { if } a \leqslant_{\mathfrak{B}(G)} b \\
-\infty & \text { if } a>_{\mathfrak{B}(G)} b=-\infty \\
b-a & \text { otherwise }\end{cases}\right.
$$

Now, using the previous functors and results from [Mon06], for each rational $c \in(0,1)_{\mathbb{Q}}$ we can construct a complete embedding $\sigma_{c}$ of each $\Pi^{\infty}$-chain $\boldsymbol{A}$ into $\mathfrak{B}\left(\mathbb{R}_{+}\right)$suitably extended with rational constants (depending on $c) .{ }^{14}$ Indeed, pick an arbitrary element $c \in(0,1)_{\mathbb{Q}}$ and let $\sigma_{c}^{\prime}: \mathfrak{G}(\boldsymbol{A}) \rightarrow \mathbb{R}_{+}$be given by $\sigma_{c}^{\prime}(0)=0, \sigma_{c}^{\prime}\left(x_{+}\right)=\sup \left\{\frac{n}{m}:\left(x_{+}\right)^{m} \geqslant\left(c_{+}^{\boldsymbol{A}}\right)^{n}\right\}$ and $\sigma_{c}^{\prime}\left(x_{-}\right)=-\sigma_{c}^{\prime}\left(x_{+}\right)$, for $x \in A, 1>x>0$. In [Mon06, Prop. 3] it is proven that this is a complete embedding. Define then the embedding $\sigma_{c}: \boldsymbol{A} \rightarrow \mathfrak{B}\left(\mathbb{R}_{+}\right)$by $\sigma_{c}(0)=-\infty, \sigma_{c}(1)=\sigma_{c}^{\prime}(0)=0$ and $\sigma_{c}(x)=\sigma_{c}^{\prime}\left(x_{-}\right)$for all $0<x<1$, and observe that it is a complete embedding too. This is immediate since $\sigma_{c}^{\prime}$ is already complete, the only case worth to be checked is whether $\sigma_{c}\left(\bigwedge_{i \in I} x_{i}\right)=\bigwedge_{i \in I} \sigma_{c}\left(x_{i}\right)$ when $\left\{x_{i}\right\}_{i \in I} \subseteq A$ is such that $x_{i}>0$ for all $i \in I$ and $\bigwedge_{i \in I} x_{i}=0$. By definition, $\sigma_{c}\left(\bigwedge_{i \in I} x_{i}\right)=-\infty$. On the other hand, if we suppose towards a contradiction that $\bigwedge_{i \in I} \sigma_{c}\left(x_{i}\right)>-\infty$, by definition we have that there is $z \in \mathbb{R}_{+}$such that $z=\bigwedge_{i \in I} \sigma_{c}^{\prime}\left(\left(x_{i}\right)_{-}\right)$. It is easy to see that, by the definition of $\sigma_{c}^{\prime}$, there exists $x_{-} \in \mathfrak{G}(\boldsymbol{A})$ such that $\sigma_{c}^{\prime}\left(x_{-}\right) \leqslant z$, and thus we get that $0<x \leqslant x_{i}$ for all $i \in I$, and so, a contradiction (since $\bigwedge_{i \in I} x_{i}=0$ ).

On the other hand, by the definition of the ordering in $\mathfrak{G}(\boldsymbol{A})$, it follows that $\left(x_{+}\right)^{m} \geqslant\left(c_{+}^{\boldsymbol{A}}\right)^{n}$ if and only if $x^{m} \leqslant\left(c^{\boldsymbol{A}}\right)^{n}$. It is direct (using that the rationals are dense in the reals, and that $c<1)$ that for any $d \in[0,1]_{\mathbb{Q}} \log _{c} d=\sup \left\{r \in \mathbb{Q}: d \leqslant c^{r}\right\}$, and so, by axiom $\left(\mathrm{A}_{\mathrm{c}} 1\right)$, we get that $\sigma_{c}\left(d^{\boldsymbol{A}}\right)=-\log _{c} d$.

Now it is a natural step to combine each one of the previously defined complete embeddings with their complementary one from $\mathfrak{B}\left(\mathbb{R}_{+}\right)$into $[0,1]_{\Pi}$ (the corresponding exponential function with base $c)$. Let $E_{c}: \mathfrak{B}\left(\mathbb{R}_{+}\right) \rightarrow[0,1]_{\Pi}$ be defined by

$$
E_{c}(x):= \begin{cases}c^{-x} & \text { if } x \in \mathbb{R}^{-} \\ 0 & \text { if } x=0\end{cases}
$$

Observe that the function $E_{c}$ is clearly a complete embedding from $\mathfrak{B}(\mathbb{R})$ into $[0,1]_{\Pi}$ (it is a continuous, monotone and increasing function since $0<c<1$ and its domain is $\mathbb{R}^{-} \cup\{0\}$ ), and $E_{c}(-1)=c$.

We can now proceed to prove that the composition $E_{c} \circ \sigma_{c}$ is a complete embedding from $\boldsymbol{A}$ into $[0,1]_{\Pi}$. Since both functions are complete embeddings, the only thing that is left to prove is that for any constant $d^{\boldsymbol{A}}$ we have that $E_{c} \circ \phi_{c}\left(d^{\boldsymbol{A}}\right)=d$. But we know that $\sigma_{c}\left(d^{\boldsymbol{A}}\right)=-\log _{c} d$, so it is direct that $E_{c}\left(\phi_{c}\left(d^{\boldsymbol{A}}\right)\right)=c^{--\log _{c} d}=d$.

Finally, it is straightforward to see that this embedding is unique.

\footnotetext{
${ }^{14}$ In the following we simplify the notation by dropping the subindices in the different order symbols since there is no danger of confusion.
} 